\title{
DYNAMIC ANALYSIS OF FRAMED STRUCTURES WITH STATISTICAL UNCERTAINTIES
}

\author{
SONDIPON ADHIKARI ${ }^{*, \dagger}$ AND C. S. MANOHAR \\ Department of Civil Engineering, Indian Institute of Science, Bangalore 560 012, India
}

\begin{abstract}
SUMMARY
The forced harmonic vibration analysis of portal frames consisting of viscously damped beams with spatial stochastic variation of mass and stiffness properties is considered. The analysis is based on the assembly of element stochastic dynamic stiffness matrices. The solution involves inversion of the global dynamic stiffness matrix, which, in this case, turns out to be a complex-valued symmetric random matrix. Three alternative approximate procedures, namely, random eigenfunction expansion method, complex Neumann expansion method and combined analytical and simulation method are used to invert the matrix. The performance of these approximate procedures is evaluated using Monte Carlo simulation results. Copyright (C) 1999 John Wiley \& Sons, Ltd.
\end{abstract}

KEY WORDS: dynamic stiffness; stochastic finite element method; random frames; random matrices; frequency response function

\section{INTRODUCTION}

Problems of linear structural dynamics have been extensively treated in the existing literature within the frameworks of finite element method using normal mode expansions, ${ }^{1}$ transfer matrix method $^{2}$ and dynamic stiffness matrix approach. ${ }^{3}$ When these methods are to be extended to analyse structures with random material and/or geometric property variations, questions on solutions of random differential and algebraic eigenvalue problems, inversion of random matrices and differential operators and characterization of random matrix products arise. This class of problems is currently receiving notable research attention. ${ }^{4-13}$ A significant development in the recent past has been the generalization of finite element method to cover the problems of static, dynamic and stability analyses of stochastically parametered systems. ${ }^{5,9-12}$

The concept of weighted integrals as a means to discretize random fields for static stochastic finite element applications has been developed earlier by Shinozuka, ${ }^{14}$ Deodatis $^{15}$ and Takada. ${ }^{16,17}$ The paper by Bucher and Brenner ${ }^{18}$ extends these formulations to dynamic applications through the use of consistent mass matrix formulation. Recently, the present authors have examined the feasibility of using frequency dependent shape functions to formulate the dynamic stiffness matrix

\footnotetext{
* Correspondence to: Sondipon Adhikari, Department of Engineering, University of Cambridge, Trumpington Street, Cambridge CB2 1PZ, U.K. E-mail: sa225@eng.cam.ac.uk

$\dagger$ Current address: Department of Engineering, University of Cambridge, Trumpington Street, Cambridge CB2 1PZ, U.K.

Contract/grant sponsor: Department of Science and Technology, Government of India
}

CCC 0029-5981/99/081157-22\$17.50

Copyright (C) 1999 John Wiley \& Sons, Ltd. 
for randomly parametered beam elements. ${ }^{19}$ This study offers an alternative to the traditional normal mode expansion method to formulate dynamics of random beam elements. The method leads to the definition of weighted integrals which are frequency dependent and which offer a powerful means to discretize random fields for dynamic applications. The major advantage here is the relief in selecting finite element mesh sizes irrespective of the frequency range of external excitation. This is in contrast with the traditional modal expansion procedures in which finer mesh is needed for modelling the low wave length vibrations associated with higher frequencies of driving.

The study reported in the present paper extends our earlier study on single beam elements ${ }^{19}$ to the problem of vibration analysis of portal frames built up of viscously damped beam elements with randomly varying mass and stiffness characteristics. A key step which arises in this study would be the inversion of a global dynamic stiffness matrix. This matrix, in the present study, has been shown to be symmetric, complex-valued and random in nature. Questions on the inverse and eigensolutions of this type of matrices appear to have received very little attention in the existing literature. The paper proposes three alternative approximate procedures for the inversion of the global stiffness matrix, namely, random eigenfunction expansion method, complex Neumann expansion method and combined analytical and simulation method. The random eigenfunction expansion method employs a Galerkin type of error minimization in a finite dimensional complex space. The second method extends the Neumann expansion technique used for finding their inverse of real valued random matrices $^{5}$ to invert the complex valued stiffness matrix. The combined analytical and simulation method employs digital simulation procedure to invert the global dynamic stiffness matrix after this matrix has been analytically formulated using frequency dependent weighted integrals. The performance of the above three approximate methods are compared with the more exact Monte Carlo simulation results. An illustrative example on the statistics of frequency response functions of a three member portal frame is presented, which brings out different features of the proposed formulations.

\section{DYNAMIC STIFFNESS MATRIX FOR A STOCHASTIC BEAM ELEMENT}

In a recent study ${ }^{19}$ we have developed a finite element based formulation to obtain the dynamic stiffness matrix of a general beam element having randomly inhomogeneous mass density, flexural and axial rigidities and elastic foundation modulus. In this section we briefly outline the procedures followed for the simpler case of a two noded random beam element having only flexural degree of freedom. Also described are the steps arising from assembly of distinct stochastic finite elements. The beam element considered in this study is shown in Figure 1. The governing field equation of motion, assuming linear behavior and the validity of the Euler-Bernoulli hypotheses, is given by

$$
\frac{\partial^{2}}{\partial x^{2}}\left[\operatorname{EI}(x) \frac{\partial^{2} Y(x, t)}{\partial x^{2}}+c_{1} \frac{\partial^{3} Y(x, t)}{\partial x^{2} \partial t}\right]+m(x) \frac{\partial^{2} Y(x, t)}{\partial t^{2}}+c_{2} \frac{\partial Y(x, t)}{\partial t}=0
$$

Here $Y(x, t)$ is the transverse flexural displacement, $\operatorname{EI}(x)$ the flexural rigidity, $m(x)$ the mass per unit length, $c_{1}$ the strain-rate-dependent viscous damping coefficient and $c_{2}$ the velocity-dependent viscous damping coefficient. The quantities $\operatorname{EI}(x)$ and $m(x)$, in this study, are modelled as meansquare bounded, homogeneous random fields and are taken to have the following form:

$$
\begin{aligned}
m(x) & =m_{0}\left[1+\varepsilon_{1} f_{1}(x)\right] \\
\mathrm{EI}(x) & =\mathrm{EI}_{0}\left[1+\varepsilon_{2} f_{2}(x)\right]
\end{aligned}
$$




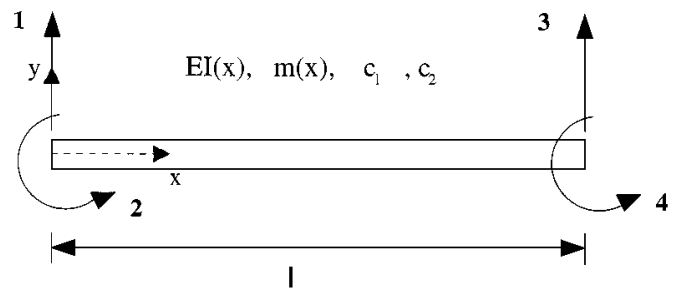

Figure 1. Random beam element in local co-ordinates

Here 0 subscript indicates the mean values, $\varepsilon_{1}$ and $\varepsilon_{2}$ are deterministic constants which are small compared to unity and the random fields $f_{1}(x)$ and $f_{2}(x)$ are taken to have zero mean, unit standard deviation with covariance $R_{i j}(\xi)=\left\langle f_{i}(x) f_{j}(x)\right\rangle(i, j=1,2)$; here $\langle\cdot\rangle$ denotes the mathematical expectation operator. Since linear system behaviour is being assumed, in steady state, the solution to the field equation can be expressed as

$$
Y(x, t)=y(x) \exp [\mathrm{i} \omega t]
$$

Consequently, the equation governing $y(x)$ has the form

$$
\frac{\mathrm{d}^{2}}{\mathrm{~d} x^{2}}\left[\operatorname{EI}(x) \frac{\mathrm{d}^{2} y}{\mathrm{~d} x^{2}}+\mathrm{i} \omega c_{1} \frac{\mathrm{d}^{2} y}{\mathrm{~d} x^{2}}\right]+\left[-m(x) \omega^{2}+c_{2} \mathrm{i} \omega\right] y=0
$$

The formulation of the dynamic stiffness matrix is achieved through a sequence of following steps.

Step 1. Derivation of shape functions: Obtain the shape functions using the deterministic undamped field equation. That is, solve the equation

$$
\frac{\mathrm{d}^{4} y}{\mathrm{~d} x^{4}}-b^{4} y=0
$$

under a set of 'binary' boundary conditions. The parameter $b$ appearing in the above equation is given by $b^{4}=m_{0} \omega^{2} / \mathrm{EI}_{0}$. The shape functions $\{\mathbf{N}(x, \omega)\}$ can be shown to be given by $\{\mathbf{N}(x, \omega)\}=[\boldsymbol{\Gamma}(\omega)]\{\mathbf{s}(x, \omega)\}$, where

$$
[\Gamma(\omega)]=\left[\begin{array}{cccc}
\frac{1}{2} \frac{c S+C s}{c C-1} & -\frac{1}{2} \frac{1+s S-c C}{c C-1} & -\frac{1}{2} \frac{c S+C s}{c C-1} & \frac{1}{2} \frac{c C+s S-1}{c C-1} \\
\frac{1}{2} \frac{c C+s S-1}{b(c C-1)} & \frac{1}{2} \frac{-C s+c S}{b(c C-1)} & -\frac{1}{2} \frac{1+s S-c C}{b(c C-1)} & -\frac{1}{2} \frac{-C s+c S}{b(c C-1)} \\
-\frac{1}{2} \frac{S+s}{c C-1} & \frac{1}{2} \frac{C-c}{c C-1} & \frac{1}{2} \frac{S+s}{c C-1} & -\frac{1}{2} \frac{C-c}{c C-1} \\
\frac{1}{2} \frac{C-c}{b(c C-1)} & -\frac{1}{2} \frac{S-s}{b(c C-1)} & -\frac{1}{2} \frac{C-c}{b(c C-1)} & -\frac{1}{2} \frac{S-s}{b(c C-1)}
\end{array}\right]
$$

and

$$
\{\mathbf{s}(x, \omega)\}=[\sin b x, \cos b x, \sinh b x, \cosh b x]^{t}
$$

is the array of basis functions. Here $C=\cosh b l, c=\cos b l, S=\sinh b l$ and $s=\sin b l$ and superscript $t$ represents matrix transpose. Plots of these shape functions, for a typical beam element to be considered later in numerical illustrations, are shown in Figures 2(a) and 2(b). It may be noted that, for the case of $\omega=0$, that is, for the static case, the shape functions are cubic polynomial in 

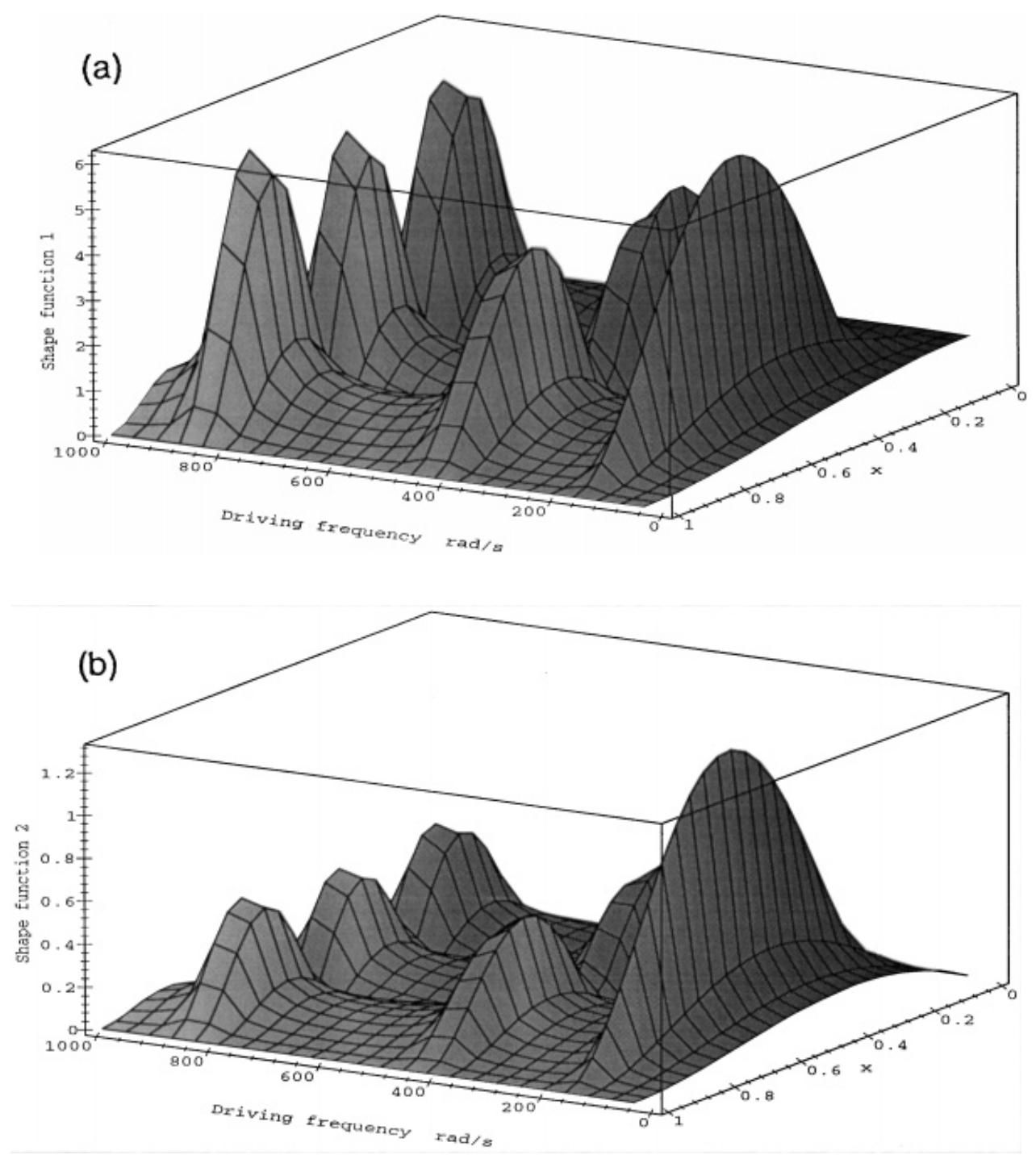

Figure 2. Shape function for element number 2 of the portal frame in Figure 3: (a) shape function 1; (b) shape function 2

$x$ and they agree with the well known beam shape functions. This feature is observable in figures 2(a) and 2(b). With increases in the value of $\omega$, the shape functions adapt themselves and herein lies their major advantage.

Step 2. Derivation of element equation of motion: The displacement field within the element is expressed in the form

$$
Y(x, t)=\sum_{j=1}^{4} d_{j}(t) N_{j}(x, \omega)
$$


Here $d_{j}(t)(j=1,4)$ are the generalized co-ordinates representing the nodal displacements. The governing equations for the generalized co-ordinates $d_{j}(t)$ can now be obtained by using the condition

$$
\frac{\mathrm{d}}{\mathrm{d} t}\left[\frac{\partial \mathscr{L}}{\partial \dot{d}_{j}}\right]-\frac{\partial \mathscr{L}}{\partial d_{j}}=0, \quad j=1,4
$$

where $\mathscr{L}(t)=T(t)-V(t)$ is the Lagrangian.

Step 3. Undamped element stiffness matrix: Since the motion is harmonic at frequency $\omega$, it follows that $d_{j}(t)=A_{j} \exp [\mathrm{i} \omega t]$. Consequently, the undamped dynamic stiffness matrix $\mathbf{D}_{\mathbf{u}}$ can be shown to be given by

$$
\mathbf{D}_{\mathrm{u}}(\omega)=\left[-\omega^{2} I_{i j}(\omega)+J_{i j}(\omega)\right]_{(4 \times 4)}
$$

with

$$
\begin{aligned}
I_{i j}(\omega) & =\int_{0}^{L} m(x) N_{i}(x, \omega) N_{j}(x, \omega) \mathrm{d} x \\
J_{i j}(\omega) & =\int_{0}^{L}\left[\operatorname{EI}(x) \frac{\mathrm{d}^{2}}{\mathrm{~d} x^{2}} N_{i}(x, \omega) \frac{\mathrm{d}^{2}}{\mathrm{~d} x^{2}} N_{j}(x, \omega)\right] \mathrm{d} x
\end{aligned}
$$

Substituting the expression of $m(x)$ and $\operatorname{EI}(x)$ from equation (2) into equations (11) and after separating the random and deterministic parts, the undamped element dynamic stiffness matrix can be written as

$$
\mathbf{D}_{\mathrm{u}}(\omega)=\overline{\mathbf{D}}_{\mathrm{u}}(\omega)+\sum_{l=1}^{10}\left[\boldsymbol{\alpha}^{l}(\omega)\right] X_{l}(\omega)
$$

In the above expression $\overline{\mathbf{D}}_{\mathrm{u}}(\omega)$ is the deterministic undamped element stiffness matrix which can be shown to be given by

$$
\overline{\mathbf{D}}_{\mathrm{u}}(\omega)=\left[\begin{array}{cccc}
\frac{\mathrm{EI}(c S+C s) b^{3}}{-1+c C} & -\frac{\mathrm{EI} s b^{2} S}{-1+c C} & \frac{\mathrm{EI}(S+s) b^{3}}{-1+c C} & -\frac{\mathrm{EI} b^{2}(C-c)}{-1+c C} \\
-\frac{\mathrm{EI} s b^{2} S}{-1+c C} & \frac{\mathrm{EI}(-C s+c S) b}{-1+c C} & \frac{\mathrm{EI} b^{2}(C-c)}{-1+c C} & -\frac{\mathrm{EI}(S-s) b}{-1+c C} \\
\frac{\mathrm{EI}(S+s) b^{3}}{-1+c C} & \frac{\mathrm{EI} b^{2}(C-c)}{-1+c C} & -\frac{\mathrm{EI}(c S+C s) b^{3}}{-1+c C} & \frac{\mathrm{EI} s b^{2} S}{-1+c C} \\
-\frac{\mathrm{EI} b^{2}(C-c)}{-1+c C} & -\frac{\mathrm{EI}(S-s) b}{-1+c C} & \frac{\mathrm{EI} s b^{2} S}{-1+c C} & \frac{\mathrm{EI}(-C s+c S) b}{-1+c C}
\end{array}\right]
$$

Furthermore, $X_{l}(l=1,10)$ are random in nature and are given by

$$
\begin{aligned}
& X_{1}=W_{11} ; X_{2}=W_{12} ; X_{3}=W_{13} ; X_{4}=W_{14} ; X_{5}=W_{22} \\
& X_{6}=W_{23} ; X_{7}=W_{24} ; X_{8}=W_{33} ; X_{9}=W_{34} ; X_{10}=W_{44}
\end{aligned}
$$

with

$$
W_{k r}=\int_{o}^{L}\left[\left\{-m_{0} \omega^{2} \varepsilon_{1} f_{1}(x)\right\} s_{k}(x) s_{r}(x)+\mathrm{EI}_{0} \varepsilon_{2} f_{2}(x) \frac{\mathrm{d}^{2} s_{k}(x)}{\mathrm{d} x^{2}} \frac{\mathrm{d}^{2} s_{r}(x)}{\mathrm{d} x^{2}}\right] \mathrm{d} x
$$

Also, $\left[\boldsymbol{\alpha}^{l}(\omega)\right](l=1,10)$ are $4 \times 4$ symmetric matrices of deterministic functions of $\omega$. Explicit expressions of the elements of these 10 matrices have been generated using symbolic manipulations 
using Maple $\mathrm{V}$ and are given in Appendix I. It may be noted that $X_{l}(\omega)(l=1,10)$, are random processes evolving in the frequency parameter $\omega$. Thus, for a fixed value of driving frequency $\omega$, these quantities are random variables and herein they are termed as Dynamic Weighted Integrals, since they arise as 'weighted integrals' of the random fields $f_{1}(x)$ and $f_{2}(x)$. It may also be noted that $X_{l}$ are linear functions of the random fields $f_{1}(x)$ and $f_{2}(x)$ and, therefore, if $f_{1}(x)$ and $f_{2}(x)$ are modeled as jointly Gaussian random fields, it follows that $X_{l}$ are also jointly Gaussian. Furthermore, since the dynamic stiffness coefficients are linear function of $X_{l}$, it follows that these coefficients in turn are also Gaussian distributed.

Step 4. Damped element stiffness matrix: To allow for the effect of damping terms present in the field equation (1) the following steps are adopted:

(a) determine the damped dynamic stiffness matrix $\mathbf{D}_{1}(\omega)$ for the deterministic homogeneous beam element, that is, $\mathbf{D}_{\mathrm{u}}(\omega)$ given by equation (13) with $\varepsilon_{1}=\varepsilon_{2}=0$ and

$$
b^{4}=\frac{m_{0} \omega^{2}+\mathrm{i} \omega c_{2}}{\mathrm{EI}_{0}+\mathrm{i} \omega c_{1}}
$$

(b) determine undamped dynamic stiffness matrix $\mathbf{D}_{2}(\omega)$ for the deterministic homogeneous beam element given again by equation (13) with $\varepsilon_{1}=\varepsilon_{2}=0$ and $b=m_{0} \omega^{2} / \mathrm{EI}_{0}$.

(c) compute the contribution to the dynamic stiffness $\mathbf{D}_{\mathrm{d}}(\omega)$ from damping terms using $\mathbf{D}_{\mathrm{d}}(\omega)=\mathbf{D}_{1}(\omega)-\mathbf{D}_{2}(\omega)$ and

(d) finally, the damped stochastic dynamic stiffness matrix is obtained as

$$
\mathbf{D}(\omega)=\mathbf{D}_{\mathrm{u}}(\omega)+\mathbf{D}_{\mathrm{d}}(\omega)
$$

Now substituting $\mathbf{D}(\omega)$ in place of $\mathbf{D}_{\mathrm{u}}(\omega)$ in equation (12) the complete element dynamic stiffness matrix for an element ' $e$ ' can be expressed as

$$
\mathbf{D}^{e}(\omega)=\overline{\mathbf{D}}^{e}(\omega)+\sum_{l=1}^{10}\left[\boldsymbol{\alpha}^{l}(\omega)\right]^{e} X_{l}^{e}
$$

Here $\overline{\mathbf{D}}^{e}(\omega)$ represents the damped deterministic element dynamics stiffness matrix of element ' $e$ '.

Step 5. Element stiffness matrix in global co-ordinates: The element stiffness matrices in global co-ordinate can be written as

$$
\mathbf{D}_{G}^{e}(\omega)=\mathbf{T}^{e^{t}} \mathbf{D}^{e}(\omega) \mathbf{T}^{e}
$$

where $\mathbf{T}^{e}$ denotes the transformation matrix for the element concerned. Now substituting expression of $\mathbf{D}^{e}(\omega)$ from equation (18) into above equation and separating the deterministic and random parts, $\mathbf{D}_{G}^{e}(\omega)$ can be cast in the form

$$
\mathbf{D}_{G}^{e}(\omega)=\overline{\mathbf{D}}_{G}^{e}(\omega)+\Delta \mathbf{D}_{G}^{e}(\omega)
$$

Here $\overline{\mathbf{D}}_{G}^{e}(\omega)=\mathbf{T}^{t} \overline{\mathbf{D}}^{e}(\omega) \mathbf{T}^{e}$ is the deterministic part of the element stiffness matrix in the global co-ordinates and $\Delta \mathbf{D}_{G}^{e}(\omega)$ is the corresponding random part. In the further analysis, the covariance matrix associated with elements of $\Delta \mathbf{D}_{G}^{e}(\omega)$ would be needed. Also required is the information 
on the cross-covariance between $\Delta \mathbf{D}_{G}^{e}(\omega)$ associated with two distinct elements $e_{u}$ and $e_{v}$. This covariance matrix is expressed as follows:

$$
\left\langle\Delta D_{G_{p q}}^{e_{u}}(\omega) \Delta D_{G_{r s}}^{e_{v}}(\omega)\right\rangle=\sum_{i=1}^{10} \sum_{j=1}^{10} \sum_{k=1}^{4} \sum_{l=1}^{4} \sum_{m=1}^{4} \sum_{n=1}^{4} T_{k p}^{e_{u}} T_{l q}^{e_{u}} T_{m r}^{e_{v}} T_{n s}^{e_{v}}\left[\alpha_{k l}^{i}\right]^{e_{u}}\left[\alpha_{m n}^{j}\right]^{e_{v}} \operatorname{Cov}\left(X_{i}^{e_{u}}, X_{j}^{e_{v}}\right)
$$

where $p, q, r, s=1,4$ and $e_{u}, e_{v}$ runs over the number of element in the structure. The term $\operatorname{Cov}\left(X_{i}^{e_{u}}, X_{j}^{e_{v}}\right)$ represents the statistics of the dynamic weighted integrals between two beam elements and is defined in the following section.

Step 6. Statistics of the dynamic weighted integrals: The covariance of the dynamic weighted integrals can be obtained by using equation (15) for two different elements of the structure, and can be expressed as

$$
\operatorname{Cov}\left(X_{i}^{e_{u}}, X_{j}^{e_{v}}\right)=\left\langle W_{k r}^{e_{u}} W_{p q}^{e_{v}}\right\rangle=\operatorname{In}_{11}+\operatorname{In}_{12}+\operatorname{In}_{22}
$$

where

$$
\begin{aligned}
\operatorname{In}_{11}= & m_{0}^{e_{u}} m_{0}^{e_{v}} \omega^{4} \varepsilon_{1}^{e_{u}} \varepsilon_{1}^{e_{v}} \int_{0}^{L_{u}} \int_{0}^{L_{v}}\left\{s_{k}^{e_{u}}\left(x_{1}\right) s_{r}^{e_{u}}\left(x_{1}\right) s_{p}^{e_{v}}\left(x_{2}\right) s_{q}^{e_{v}}\left(x_{2}\right) R_{11}^{e_{u} e_{v}}\left(x_{1}, x_{2}\right)\right\} \mathrm{d} x_{1} \mathrm{~d} x_{2} \\
\operatorname{In}_{12}= & -\omega^{2} \varepsilon_{1}^{e_{u}} \varepsilon_{2}^{e_{v}} \int_{0}^{L_{u}} \int_{0}^{L_{v}}\left\{m_{0}^{e_{u}} \mathrm{EI}_{0}^{e_{v}} s_{k}^{e_{u}}\left(x_{1}\right) s_{r}^{e_{u}}\left(x_{1}\right) \frac{\mathrm{d}^{2} s_{p}^{e_{v}}\left(x_{2}\right)}{\mathrm{d} x_{2}^{2}} \frac{\mathrm{d}^{2} s_{q}^{e_{v}}\left(x_{2}\right)}{\mathrm{d} x_{2}^{2}}\right. \\
& \left.+m_{0}^{e_{v}} \mathrm{EI}_{0}^{e_{u}} \frac{\mathrm{d}^{2} s_{k}^{e_{u}}\left(x_{1}\right)}{\mathrm{d} x_{1}^{2}} \frac{\mathrm{d}^{2} s_{r}^{e_{u}}\left(x_{1}\right)}{\mathrm{d} x_{1}^{2}} s_{p}^{e_{v}}\left(x_{2}\right) s_{q}^{e_{v}}\left(x_{2}\right)\right\} R_{12}^{e_{u} e_{v}}\left(x_{1}, x_{2}\right) \mathrm{d} x_{1} \mathrm{~d} x_{2} \\
\operatorname{In}_{22}= & \operatorname{EI}_{0}^{e_{u}} \operatorname{EI}_{0}^{e_{v}} \varepsilon_{2}^{e_{u}} \varepsilon_{2}^{e_{v}} \int_{0}^{L_{u}} \int_{0}^{L_{v}}\left\{\frac{\mathrm{d}^{2} s_{k}^{e_{u}}\left(x_{1}\right)}{\mathrm{d} x_{1}^{2}} \frac{\mathrm{d}^{2} s_{r}^{e_{u}}\left(x_{1}\right)}{\mathrm{d} x_{1}^{2}} \frac{\mathrm{d}^{2} s_{p}^{e_{v}}\left(x_{2}\right)}{\mathrm{d} x_{2}^{2}} \frac{\mathrm{d}^{2} s_{q}^{e_{v}}\left(x_{2}\right)}{\mathrm{d} x_{2}^{2}} R_{22}^{e_{u} e_{v}}\left(x_{1}, x_{2}\right)\right\} \mathrm{d} x_{1} \mathrm{~d} x_{2}
\end{aligned}
$$

In the above equations, $i, j=1,10$; the relationship between $i, j$ and $k, r, p, q$ is according to equation (14), $L_{u}, L_{v}=$ lengths of the elements $e_{u}$ and $e_{v}$ respectively and (.) $)^{e_{u}}$ represents the properties (.) corresponding to the element $e_{u}$. The expression $R_{l m}^{e_{u} e_{v}}\left(x_{1}, x_{2}\right),(l, m=1,2)$ appearing in the above equations denotes the covariance function of the random processes $f_{l}\left(x_{1}\right)$ and $f_{m}\left(x_{2}\right)$ between the elements $e_{u}$ and $e_{v}$, that is $R_{l m}^{e_{u} e_{v}}\left(x_{1}, x_{2}\right)=\left\langle f_{l}^{e_{u}}\left(x_{1}\right) f_{m}^{e_{v}}\left(x_{2}\right)\right\rangle$. Thus it can be seen that the random variability in the dynamic stiffness coefficients of the beam element gets characterized completely in terms of the dynamic weighted integrals.

In certain situations it may be advantageous to linearly transform the weighted integrals such that in the transformed space they become mutually uncorrelated. As is well known, the matrix of eigenvectors of the covariance matrix $\operatorname{Cov}\left(X_{i}^{e_{u}}, X_{j}^{e_{v}}\right)$ serves as the transformation matrix to achieve this goal. In the present study, however, it was observed in the numerical work that this type of transformation, although proved to be advantageous for low frequencies, did not offer significant advantage over the driving frequency range considered.

\section{GLOBAL DYNAMIC STIFFNESS MATRIX}

After having obtained the element dynamic stiffness matrices in the global co-ordinate system, these matrices can further be assembled to derive the global dynamic stiffness matrix. The rules 
for assembling the element stiffness matrices are identical to those used in the traditional static finite element analysis. This leads to the expression

$$
\mathbf{K}_{\mathbf{G}}(\omega)=\sum_{e=1}^{n e} \mathbf{D}_{G}^{e}(\omega)
$$

where $\mathbf{K}_{\mathbf{G}}(\omega)$ is the global dynamic stiffness matrix, ne the number of elements and $\mathbf{D}_{G}^{e}(\omega)$ the element dynamic stiffness matrix in the global co-ordinate system. The summation here implies the addition of appropriate element stiffness matrices at appropriate locations within the global stiffness matrix. The reduced global stiffness matrix $\mathbf{K}(\omega)$ can now be obtained by deleting the rows and columns of $\mathbf{K}_{\mathbf{G}}(\omega)$ corresponding to the fixed degrees of freedom. The equation of equilibrium is given by

$$
\mathbf{K}(\omega) \mathbf{Z}(\omega)=\mathbf{F}
$$

where $\mathbf{Z}(\omega)$ is the amplitude of the nodal harmonic displacement vector to be determined and $\mathbf{F}$ is the amplitude of nodal harmonic force vector and $\omega$ is the driving frequency. The reduced global dynamic stiffness matrix can further be written as

$$
\mathbf{K}(\omega)=\mathbf{K}^{0}(\omega)+\Delta \mathbf{K}(\omega)
$$

where $\mathbf{K}^{0}(\omega)$ is the deterministic part and $\Delta \mathbf{K}(\omega)$ is the stochastic part. The deterministic part of the matrix is observed to be complex valued and symmetric in nature while the stochastic part is real valued and symmetric. The latter feature arises here because in our study

(a) element damping terms are taken to be deterministic and

(b) we have used shape functions which are independent of damping terms.

The deterministic part is further represented by

$$
\mathbf{K}^{0}(\omega)=\left[\mathbf{K}_{\mathrm{R}}^{0}(\omega)+\mathrm{i} \mathbf{K}_{\mathrm{I}}^{0}(\omega)\right]
$$

where $\mathbf{K}_{\mathrm{R}}^{0}(\omega)$ and $\mathbf{K}_{\mathrm{I}}^{0}(\omega)$ are, respectively, the real and imaginary parts of $\mathbf{K}^{0}(\omega)$. As has been already pointed out, when element property random fields arise as Gaussian fields, the elements of stochastic part of the dynamic element stiffness matrix, in the present approximation, also become Gaussian distributed. Furthermore, since the global dynamic stiffness matrix is obtained by linear superpositioning of element stiffness matrices, see equation (24), it follows that elements of $\Delta \mathbf{K}(\omega)$ are also Gaussian distributed. Thus, the complete description of $\mathbf{K}(\omega)$ is given by its mean $\mathbf{K}^{0}(\omega)$ and the covariance matrix of the weighted integrals associated with the ne number of finite elements. It may be pointed here that the formulation presented here is capable of accounting for cross-correlations among system property random fields associated with distinct finite elements.

\section{INVERSION OF GLOBAL DYNAMIC STIFFNESS MATRIX}

The problem of determination of $\mathbf{Z}(\omega)$ requires the inversion of the global dynamic stiffness matrix. From the discussion presented in the previous section it has emerged that, according to the present formulation, elements of $\mathbf{K}(\omega)$, for a fixed value of $\omega$, are complex valued Gaussian random variables. Upon inversion, however, elements of $\mathbf{K}^{-1}(\omega)$ become, in general, non-Gaussian, which, consequently, makes $\mathbf{Z}(\omega)$ non-Gaussian. Furthermore, since $\mathbf{Z}(\omega)$ is complex valued, it 
can be described either in terms of its real and imaginary parts, or alternatively, one needs to find the associated amplitude and phase vectors. In the latter case a further nonlinear transformation of real and imaginary parts of $\mathbf{Z}(\omega)$ is implied. Thus, even to find simple descriptors of the solution, such as mean and standard deviation, the knowledge of the joint probability density function of real and imaginary parts of $\mathbf{Z}(\omega)$ is needed. This difficulty basically arises because of the complex nature of $\mathbf{Z}(\omega)$ which, in turn, arises because of damping terms. In this aspect, vibration problems of undamped systems and static problems can be considered to be relatively simpler. In the subsequent sections we present three alternative strategies to find the inverse of $\mathbf{K}(\omega)$. The first two approaches are analytical in nature while the last employs simulation techniques. In developing the analytical methods, we have introduced assumptions in such a way that the real and imaginary parts of the solution vector, for fixed $\omega$, become jointly Gaussian. This will enable us to evaluate the associated joint probability density function of real and imaginary parts of $\mathbf{Z}(\omega)$ and, hence, the statistics of the associated amplitude and phase.

\subsection{Random eigenfunction expansion method}

The equation of equilibrium (25) represents a set of linear random algebraic equations in $\mathbf{Z}(\omega)$. We seek an approximate solution to this equation of the form

$$
\hat{\mathbf{Z}}=[\boldsymbol{\Phi}]\{\mathbf{a}\}
$$

where $\boldsymbol{\Phi}$ represents a set of random basis vectors with known joint statistics and a is a vector of unknown complex valued deterministic constants. These unknown constants are determined by adopting a Galerkin type of error minimization scheme ${ }^{20}$ which leads to the expressions for the unknown $\mathbf{a}$ in terms of the statistics of elements of $\boldsymbol{\Phi}$. In principle any set of basis functions can be chosen to represent the unknown $\mathbf{Z}(\omega)$ : in this study, these functions are taken to be the eigenvectors of the real part of the reduced global dynamic stiffness matrix. That is, the basis vectors are taken to be the eigenvectors of the matrix $\mathbf{K}_{R}(\omega)=\mathbf{K}_{\mathrm{R}}^{0}(\omega)+\Delta \mathbf{K}(\omega)$. It may be noted that this matrix is symmetric, real valued, positive definite and its elements form a set of Gaussian random variables. The statistics of the eigenvectors of this matrix can be determined by solving the random eigenvalue problem given by

$$
\left[\mathbf{K}_{\mathrm{R}}^{0}(\omega)+\Delta \mathbf{K}(\omega)\right] \phi=\lambda \phi
$$

This type of random eigenvalue problems typically arise in the determination of natural frequencies and buckling loads of randomly parametered structures and has attracted the attention of many researches in the past. ${ }^{4,7,10,21}$ The analysis based on perturbation approach has received notable attention. ${ }^{21}$ We borrow these earlier results and adopt them to derive a suitable set of basis eigenvectors. It may be pointed out that, the random eigensolutions that are being used in this study do not have any direct physical meaning. The details of the perturbation method that has been used for determining the statistics of the random basis vectors is briefly outlined in Appendix II.

To determine the unknown constant $\mathbf{a}$, we begin by substituting $\hat{\mathbf{Z}}$ in equation (25) and define the error vector $\{\xi\}$ given by

$$
\{\xi\}_{(n \times 1)}=\mathbf{K}(\omega)[\boldsymbol{\Phi}]\{\mathbf{a}\}-\mathbf{F}=\left[\mathbf{K}_{\mathrm{R}}(\omega)+\mathrm{i} \mathbf{K}_{\mathrm{I}}^{0}(\omega)\right][\mathbf{\Phi}]\{\mathbf{a}\}-\mathbf{F}
$$

Adopting the Galerkin weighted residual method, we impose the conditions

$$
\left\langle\left({ }^{j} \phi, \xi\right)\right\rangle=0 ; \quad \text { for } j=1, n
$$


where (.,.) defines the inner product norm and ${ }^{j} \phi$ is the $j$ th column of matrix $\boldsymbol{\Phi}$ which plays the role of the weighting function. This equation can be recast in a matrix form as

$$
\left\langle[\Phi]^{\mathrm{t}}\{\xi\}\right\rangle=0
$$

Substituting $\{\xi\}$ from equation (30) into the above equation we obtain

$$
\left[\left\langle\boldsymbol{\Phi}^{\mathrm{t}} \mathbf{K}_{\mathrm{R}}(\omega) \boldsymbol{\Phi}\right\rangle+\mathrm{i}\left\langle\boldsymbol{\Phi}^{\mathrm{t}} \mathbf{K}_{\mathrm{I}}^{0}(\omega) \boldsymbol{\Phi}\right\rangle\right]\{\mathbf{a}\}=\left\langle\boldsymbol{\Phi}^{\mathrm{t}} \mathbf{F}\right\rangle
$$

which leads to

$$
\left[[\bar{\lambda}]+\mathrm{i}\left\langle\boldsymbol{\Phi}^{\mathrm{t}} \mathbf{K}_{\mathrm{I}}^{0}(\omega) \boldsymbol{\Phi}\right\rangle\right]\{\mathbf{a}\}=\left\langle\boldsymbol{\Phi}^{\mathrm{t}}\right\rangle \mathbf{F}
$$

The above equation can further be written concisely as

$$
\mathbf{G}\{\mathbf{a}\}=\mathbf{P}
$$

where

$$
\mathbf{G}=[\bar{\lambda}]+\mathrm{i}\left\langle\boldsymbol{\Phi}^{\mathrm{t}} \mathbf{K}_{\mathrm{I}}^{0}(\omega) \boldsymbol{\Phi}\right\rangle \quad \text { and } \quad \mathbf{P}=\left\langle\boldsymbol{\Phi}^{\mathrm{t}}\right\rangle \mathbf{F}
$$

Here $[\bar{\lambda}]$ is a diagonal matrix containing the mean eigenvalues. Now the matrix $\mathbf{G}$ in the above equation can be written in index form as

$$
G_{i j}=\delta_{i j} \bar{\lambda}_{i}+\mathrm{i} \bar{w}_{i j}
$$

where $\delta_{i j}$ is the Kronecker delta function and $\bar{w}_{i j}$ is given by

$$
\bar{w}_{i j}=\left\langle w_{i j}\right\rangle=\sum_{r=1}^{n} \sum_{s=1}^{n}\left\langle\phi_{r i} \phi_{s j}\right\rangle K_{\mathrm{I}_{r s}}^{0}
$$

The term $\left\langle\phi_{r i} \phi_{s j}\right\rangle$ in the above equation represents the correlation between $r$ th element of $i$ th eigenvector and $s$ th element of $j$ th eigenvector; see Appendix II for the details of derivation of this quantity. This term is given by

$$
\begin{aligned}
\left\langle\phi_{r i} \phi_{s j}\right\rangle= & \sum_{l=1}^{n} \sum_{m=1}^{n} C_{r l} C_{s m}\left[\left(1-\delta_{l i}\right)\left(1-\delta_{m j}\right) \frac{1}{\mu_{l i} \mu_{m j}} \sum_{p=1}^{n} \sum_{q=1}^{n} \sum_{t=1}^{n} \sum_{k=1}^{n} C_{p l} C_{q i} C_{t m} C_{k j}\right. \\
& \left.\times\left\langle\Delta K_{p q}(\omega) \Delta K_{t k}(\omega)\right\rangle\right]
\end{aligned}
$$

Here $\mu_{i j}=\mu_{i}-\mu_{j}$ and $\mu_{i}$ denotes the eigenvalues of the real deterministic matrix $\mathbf{K}_{\mathrm{R}}^{0}(\omega), C_{i j}$ are the component of $\mathbf{C}$ which is the matrix containing the normalized eigenvector of $\mathbf{K}_{\mathrm{R}}^{0}(\omega)$. The quantity $\left\langle\Delta K_{p q}(\omega) \Delta K_{t k}(\omega)\right\rangle$ denotes the correlations between the stiffness coefficients which in turn are expressible in terms of the statistics of the weighted integrals. Thus, having determined the matrix $\mathbf{G}$, the unknown constants a can now be determined using equation (34) which further leads to

$$
\mathbf{Z}(\omega)=[\boldsymbol{\Phi}]\{\mathbf{a}\}
$$

Furthermore, from the solution of random eigenvalue problem outlined in Appendix II, it is to be noted that the elements of $[\boldsymbol{\Phi}]$, that is, ${ }^{j} \phi(j=1, n)$ as determined by a first order perturbation solution, are Gaussian distributed. From equation (38) it further follows that, in the present 
approximation, the solution vector $\mathbf{Z}(\omega)$, for a fixed value of $\omega$, is also a vector of Gaussian random variables. This feature facilitates the evaluation of the statistics of the amplitude and phase vectors associate with $\mathbf{Z}(\omega)$. To achieve this, we separate $\mathbf{Z}(\omega)$ and a into their respective real and imaginary parts and write

$$
z_{i}=z_{i}^{\mathrm{R}}+\mathrm{i} z_{i}^{\mathrm{I}} \quad \text { where } z_{i}^{\mathrm{R}}=\sum_{j=1}^{n} \phi_{i j} a_{j}^{\mathrm{R}} \quad \text { and } \quad z_{i}^{\mathrm{I}}=\sum_{j=1}^{n} \phi_{i j} a_{j}^{\mathrm{I}}
$$

with

$$
\begin{aligned}
\left\langle z_{i}^{\mathrm{R}}\right\rangle & =\sum_{j=1}^{n} C_{i j} a_{j}^{\mathrm{R}} ; \quad\left\langle z_{i}^{\mathrm{I}}\right\rangle=\sum_{j=1}^{n} C_{i j} a_{j}^{\mathrm{I}} \\
\left\langle z_{i}^{\mathrm{R}}{ }^{2}\right\rangle & =\sum_{j=1}^{n} \sum_{k=1}^{n}\left\langle\phi_{i j} \phi_{i k}\right\rangle a_{j}^{\mathrm{R}} a_{k}^{\mathrm{R}} ; \quad\left\langle z_{i}^{\mathrm{I}^{2}}\right\rangle=\sum_{j=1}^{n} \sum_{k=1}^{n}\left\langle\phi_{i j} \phi_{i k}\right\rangle a_{j}^{\mathrm{I}} a_{k}^{\mathrm{I}} \\
\left\langle z_{i}^{\mathrm{R}} z_{i}^{\mathrm{I}}\right\rangle & =\sum_{j=1}^{n} \sum_{k=1}^{n}\left\langle\phi_{i j} \phi_{i k}\right\rangle a_{j}^{\mathrm{R}} a_{k}^{\mathrm{I}}
\end{aligned}
$$

In the above expressions $(.)^{\mathrm{R}}$ and $(.)^{\mathrm{I}}$ are, respectively, the real and imaginary part of (.). It may be noted that the second order statistics of $z_{i}^{\mathrm{R}}$ and $z_{i}^{\mathrm{I}}$ are again dependent upon joint statistics of the basis random vectors. Subsequently the moments of amplitude and phase of the elements of $\mathbf{Z}(\omega)$ are given by

$$
\begin{aligned}
& \left\langle\left|z_{i}\right|\right\rangle=\int_{-\infty}^{\infty} \int_{-\infty}^{\infty} \sqrt{z_{i}^{\mathrm{R}}+z_{i}^{\mathrm{I}^{2}}} p_{z_{i}^{\mathrm{R}} z_{i}^{\mathrm{I}}}\left(z_{i}^{\mathrm{R}}, z_{i}^{\mathrm{I}}\right) \mathrm{d} z_{i}^{\mathrm{R}} \mathrm{d} z_{i}^{\mathrm{I}} \\
& \left\langle\left|z_{i}\right|^{2}\right\rangle=\int_{-\infty}^{\infty} \int_{-\infty}^{\infty}\left(z_{i}^{\mathrm{R}^{2}}+z_{i}^{\mathrm{I}^{2}}\right) p_{z_{i}^{\mathrm{R}} z_{i}^{\mathrm{I}}}\left(z_{i}^{\mathrm{R}}, z_{i}^{\mathrm{I}}\right) \mathrm{d} z_{i}^{\mathrm{R}} \mathrm{d} z_{i}^{\mathrm{I}} \\
& \left\langle\left(\arg \left(z_{i}\right)\right\rangle=\int_{-\infty}^{\infty} \int_{-\infty}^{\infty}\left\{\tan ^{-1}\left(\frac{z_{i}^{\mathrm{I}}}{z_{i}^{\mathrm{R}}}\right)\right\} p_{z_{i}^{\mathrm{R}} z_{i}^{\mathrm{I}}}\left(z_{i}^{\mathrm{R}}, z_{i}^{\mathrm{I}}\right) \mathrm{d} z_{i}^{\mathrm{R}} \mathrm{d} z_{i}^{\mathrm{I}}\right. \\
& \left\langle\left(\arg \left(z_{i}\right)\right)^{2}\right\rangle=\int_{-\infty}^{\infty} \int_{-\infty}^{\infty}\left\{\tan ^{-1}\left(\frac{z_{i}^{\mathrm{I}}}{z_{i}^{\mathrm{R}}}\right)\right\}^{2} p_{z_{i}^{\mathrm{R}}} z_{i}^{\mathrm{I}}\left(z_{i}^{\mathrm{R}}, z_{i}^{\mathrm{I}}\right) \mathrm{d} z_{i}^{\mathrm{R}} \mathrm{d} z_{i}^{\mathrm{I}}
\end{aligned}
$$

In the above equations $p_{z_{i}^{\mathrm{R}} z_{i}^{\mathrm{I}}}\left(z_{i}^{\mathrm{R}}, z_{i}^{\mathrm{I}}\right)$ is the two-dimensional joint probability density function of $z_{i}^{\mathrm{R}}$ and $z_{i}^{\mathrm{I}}$. This is completely characterized by the mean, standard deviation and correlation coefficient of $z_{i}^{\mathrm{R}}$ and $z_{i}^{\mathrm{I}}$ which can be obtained from equations (40).

\subsection{Complex Neumann expansion method}

Neumann expansion method as applied to the inversion of static stiffness matrix in stochastic finite element applications has been discussed by Shinozuka and Yamazaki. ${ }^{5}$ Here we extend this formulation to invert complex-valued dynamic stiffness matrices. For this purpose we begin by writing the equilibrium equation in the following form:

$$
\left[\mathbf{K}_{\mathrm{R}}^{0}(\omega)+\mathrm{i} \mathbf{K}_{\mathrm{I}}^{0}(\omega)+\Delta \mathbf{K}(\omega)\right] \mathbf{Z}(\omega)=\mathbf{F}
$$

Let $\mathbf{Z}^{0}(\omega)$ denote the solution in absence of system randomness. This is given by the solution of the equation

$$
\mathbf{K}^{0}(\omega) \mathbf{Z}^{0}(\omega)=\mathbf{F}
$$


where $\mathbf{K}^{0}(\omega)=\left[\mathbf{K}_{\mathrm{R}}^{0}(\omega)+\mathrm{i} \mathbf{K}_{\mathrm{I}}^{0}(\omega)\right]$ is the deterministic damped dynamic stiffness matrix. According to the Neumann expansion we have

$$
\mathbf{Z}(\omega)=\left[\mathbf{K}^{0}(\omega)+\Delta \mathbf{K}(\omega)\right]^{-1} \mathbf{F}=\left[\mathbf{I}-\mathbf{R}+\mathbf{R}^{\mathbf{2}}-\mathbf{R}^{\mathbf{3}}+\cdots\right] \mathbf{Z}^{0}(\omega)
$$

with

$$
\mathbf{R}=\mathbf{K}^{0^{-1}}(\omega) \Delta \mathbf{K}(\omega)=\left[\mathbf{K}_{\mathrm{R}}^{0}(\omega)+\mathrm{i} \mathbf{K}_{\mathrm{I}}^{0}(\omega)\right]^{-1} \Delta \mathbf{K}(\omega)
$$

and $\mathbf{I}$ is the unit matrix. It must be noted that $\mathbf{R}$ is complex-valued and is random in nature. Since, in the present method of random filed discretization, the elements of $\Delta \mathbf{K}(\omega)$ are Gaussian distributed, it follows that elements of real and imaginary parts of $\mathbf{R}$ are also Gaussian distributed. From equation (45), we notice that, if only the first order terms are retained in the series expansion, the response vector $\mathbf{Z}(\omega)$ also has Gaussian distributed elements. Thus, retaining only the linear terms in $\mathbf{R}$, the response vector $\mathbf{Z}(\omega)$ is given by

$$
\mathbf{Z}(\omega)=[\mathbf{I}-\mathbf{R}] \mathbf{Z}^{0}(\omega) \quad \text { or } \quad z_{i}=z_{i}^{o}-\sum_{j=1}^{n} R_{i j} z_{j}^{o}
$$

Now, for notational convenience, we write $\mathbf{Q}=\mathbf{K}_{0}^{-1}(\omega)$, and consequently equation (46) can be rewritten as

$$
\mathbf{R}=\mathbf{Q} \Delta \mathbf{K}(\omega) \quad \text { or } \quad R_{i j}=\sum_{l=1}^{n} Q_{i l} \Delta K_{l j}
$$

Furthermore, substituting $R_{i j}$ from equation (48) into equation (47), the elements of the solution vector can be written as

$$
z_{i}=z_{i}^{0}-\sum_{j=1}^{n} \sum_{l=1}^{n} Q_{i l} z_{j} \Delta K_{l j}
$$

These elements are complex quantities with real and imaginary parts being Gaussian distributed. Denoting by $z_{i}^{\mathrm{R}}$ and $z_{i}^{\mathrm{I}}$ the real and imaginary parts of $z_{i}$, respectively, we get

$$
\begin{aligned}
& z_{i}^{\mathrm{R}}=z_{i}^{0^{\mathrm{R}}}-\sum_{j_{1}=1}^{n} \sum_{l_{1}=1}^{n}\left(Q_{i l_{1}}^{\mathrm{R}} z_{j_{1}}^{0^{\mathrm{R}}}-Q_{i l_{1}}^{\mathrm{I}} z_{j_{1}}^{0^{\mathrm{I}}}\right) \Delta K_{l_{1} j_{1}} \\
& z_{i}^{\mathrm{I}}=z_{i}^{0^{\mathrm{I}}}-\sum_{j_{2}=1}^{n} \sum_{l_{2}=1}^{n}\left(Q_{i l_{2}}^{\mathrm{I}} z_{j_{2}}^{0^{\mathrm{R}}}+Q_{i l_{2}}^{\mathrm{R}} z_{j_{2}}^{0^{\mathrm{I}}}\right) \Delta K_{l_{2} j_{2}}
\end{aligned}
$$

These expressions are further simplified to get

$$
z_{i}^{\mathrm{R}}=z_{i}^{0^{\mathrm{R}}}-U_{i}^{\mathrm{R}} ; \quad z_{i}^{\mathrm{I}}=z_{i}^{0^{\mathrm{I}}}-U_{i}^{\mathrm{I}}
$$

with

$$
\begin{aligned}
\left\langle z_{i}^{\mathrm{R}}\right\rangle & =z_{i}^{0^{\mathrm{R}}}, \quad\left\langle z_{i}^{\mathrm{I}}\right\rangle=z_{i}^{0^{\mathrm{I}}} \\
\operatorname{Var}\left(z_{i}^{\mathrm{R}}\right) & =\left\langle U_{i}^{\mathrm{R}^{2}}\right\rangle \\
& =\sum_{j_{1}=1}^{n} \sum_{l_{1}=1}^{n} \sum_{j_{2}=1}^{n} \sum_{l_{2}=1}^{n}\left(Q_{i l_{1}}^{\mathrm{R}} z_{j_{1}}^{0^{\mathrm{R}}}-Q_{i l_{1}}^{\mathrm{I}} z_{j_{1}}^{0^{\mathrm{I}}}\right)\left(Q_{i l_{2}}^{\mathrm{R}} z_{j_{2}}^{0^{\mathrm{R}}}-Q_{i l_{2}}^{\mathrm{I}} z_{j_{2}}^{0^{\mathrm{I}}}\right)\left\langle\Delta K_{l_{1} j_{1}} \Delta K_{l_{2} j_{2}}\right\rangle
\end{aligned}
$$




$$
\begin{aligned}
\operatorname{Var}\left(z_{i}^{\mathrm{I}}\right) & =\left\langle U_{i}^{\mathrm{I}^{2}}\right\rangle \\
& =\sum_{j_{1}=1}^{n} \sum_{l_{1}=1}^{n} \sum_{j_{2}=1}^{n} \sum_{l_{2}=1}^{n}\left(Q_{i l_{1}}^{\mathrm{I}} z_{j_{1}}^{0^{\mathrm{R}}}+Q_{i l_{1}}^{\mathrm{R}} z_{j_{1}}^{0^{\mathrm{I}}}\right)\left(Q_{i l_{2}}^{\mathrm{I}} z_{j_{2}}^{0^{\mathrm{R}}}+Q_{i l_{2}}^{\mathrm{R}} z_{j_{2}}^{0^{\mathrm{I}}}\right)\left\langle\Delta K_{l_{1} j_{1}} \Delta K_{l_{2} j_{2}}\right\rangle
\end{aligned}
$$

and

$$
\begin{aligned}
\operatorname{Cov}\left(z_{i}^{\mathrm{R}} z_{i}^{\mathrm{I}}\right) & =\left\langle U_{i}^{\mathrm{R}} U_{i}^{\mathrm{I}}\right\rangle \\
& =\sum_{j_{1}=1}^{n} \sum_{l_{1}=1}^{n} \sum_{j_{2}=1}^{n} \sum_{l_{2}=1}^{n}\left(Q_{i l_{1}}^{\mathrm{R}} z_{j_{1}}^{0^{\mathrm{R}}}-Q_{i l_{1}}^{\mathrm{I}} z_{j_{1}}^{0^{\mathrm{I}}}\right)\left(Q_{i l_{2}}^{\mathrm{I}} z_{j_{2}}^{0^{\mathrm{R}}}+Q_{i l_{2}}^{\mathrm{R}} z_{j_{2}}^{0^{\mathrm{I}}}\right)\left\langle\Delta K_{l_{1} j_{1}} \Delta K_{l_{2} j_{2}}\right\rangle
\end{aligned}
$$

As has been noted already, $z_{i}^{\mathrm{R}}$ and $z_{i}^{\mathrm{I}}$, for a fixed value of $\omega$, are Gaussian distributed random variables. Thus, using the above expressions, the joint probability density function of $z_{i}^{\mathrm{R}}$ and $z_{i}^{\mathrm{I}}$ can be derived. Consequently, the moments of amplitude and phase of $z_{i}$ can further be evaluated using equations (41) and (42).

\subsection{Combined analytical and simulation method}

The analytical methods presented in the preceding two sections introduce approximations at the stage of inverting the random dynamic stiffness matrix. These approximations are in addition to those involved in discretizing the random fields to formulate the element stiffness matrices and in handling damping properties. The approximations associated with inverting the matrix can be avoided if one adopts Monte Carlo simulation strategy to invert the reduced global stiffness matrix. This would require

(a) digital simulation of samples of $\mathbf{\Delta K}(\omega)$; as has been already noted, in the present approximation, the elements of $\Delta \mathbf{K}(\omega)$, for fixed $\omega$, are a set of Gaussian distributed random variables and these can be easily simulated;

(b) simulation of samples of $\mathbf{Z}(\omega)$ by numerically inverting $\mathbf{K}(\omega)$ and

(c) statistical processing of samples of $\mathbf{Z}(\omega)$ to arrive at the required statistics of amplitude and phase of $\mathbf{Z}(\omega)$.

Thus, this method treats the inversion of the stiffness matrix in an exact manner while the other steps in the solution continue to be approximate in nature. The source of approximation here is associated with the discretization of the random fields, treatment of damping and also with the use of limited number of samples for estimating the response statistics. An advantage of this method over the other two methods presented so far is that it leads to non-Gaussian estimates for elements of $\mathbf{Z}(\omega)$.

\section{FULL-SCALE DIGITAL SIMULATIONS}

Given the approximate nature of the methods presented in the previous section, it is essential that their performance be assessed by comparing the results obtained using these methods with those from more exact procedures. Such procedures can be formulated by combining the Monte Carlo simulation procedures with traditional normal mode expansion based finite element procedures, transfer matrix techniques or direct dynamic stiffness matrix approach. In this study we resort to 
the last mentioned approach. This consists of the following steps:

1. Discretize the given structure into as many elements as is the number of beams: thus, a three leg portal frame gets discretized into three elements.

2. Digital simulation of samples of $\operatorname{EI}(x)$ and $m(x)$ for all the elements considered.

3. Derivation of sample dynamic stiffness matrix for each of the elements; this requires the harmonic response analysis of inhomogeneous beam elements; the procedure described by Manohar and Adhikari ${ }^{19}$ and Sarkar and Manohar ${ }^{20}$ has been used for this purpose; this is based on converting the governing boundary value problems into a set of equivalent initial value problems and integrating these resulting equations numerically using Runge-Kutta algorithm; this leads to solutions which are 'exact' within the framework of accuracy of Runge-Kutta algorithms.

4. Formulation of sample global stiffness matrix and its numerical inversion leading to sample solution vectors.

5. Statistical processing of the ensemble of response vectors to obtain the desire statistics

Thus, in this approach, the three major steps of the response analysis, namely, discretization of random fields, treatment of damping terms and inversion of random stiffness matrix, are all handled in an exact manner with the only source of approximation being the finite size of samples used to estimate the response statistics. In terms of computational effort needed, this method obviously is more demanding than the three methods discussed in Section 4.

\section{NUMERICAL RESULTS AND DISCUSSION}

To illustrate the relative performance of the different formulations developed in this study, we consider the harmonic response analysis of a three-leg portal frame shown in Figure 3. The flexural rigidity and mass density for each element are taken to be independent, homogeneous, Gaussian random fields. It is assumed that $\varepsilon_{1}=\varepsilon_{2}=0.05$, and the autocovariance of the processes $f_{1}(x)$ and $f_{2}(x)$ for all the three beam elements are taken to be of the form

$$
R_{i i}(\xi)=\cos \lambda_{i} \xi, \quad i=1,2
$$

with $\lambda_{i}=10 \pi$ per unit length. The particular choice of autocovariance function and its parameters in this example is made only for purpose of illustrations and the theoretical results developed are expected to be valid for other forms of these functions also. It is also assumed that the random properties of distinct elements are mutually uncorrelated. It may be recalled that the harmonic displacement amplitudes for damped structural systems are complex valued. When the structural element is random, the displacement amplitudes can be interpreted as complex valued random processes evolving in the frequency parameter $\omega$. In this study we focus our attention on evaluating the mean and standard deviation of the amplitude and phase of the side sway as a function of the driving frequency $\omega$. The results of theoretical analysis are compared with a 100 samples full-scale Monte Carlo simulation in Figures 4-8. The algorithm used in the simulation work to simulate samples of the dynamic element stiffness matrix is as outlined in our earlier works. ${ }^{19,20}$ It must be emphasized that the simulation work involves no discretization of the random fields, it treats the damping terms appearing in equation (1) exactly and, also, it inverts the random complex matrix in an exact way. Therefore, the simulation results serve to evaluate several aspects of the approximate analytical procedures. All programs developed for the purpose of this study 


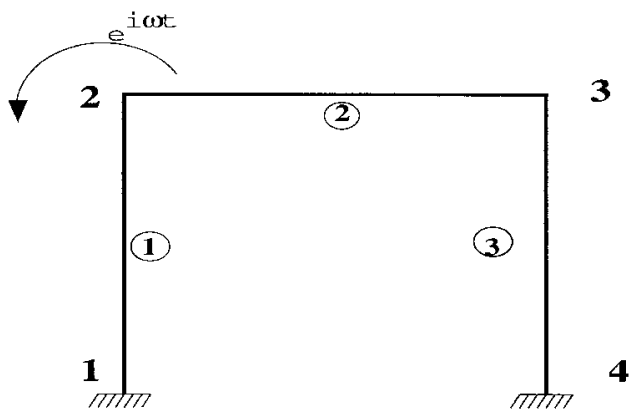

Figure 3. Finite element model for dynamic stiffness analysis of portal frame; $E I_{0}=10 \cdot 0, L=1 \cdot 0, m_{0}=0 \cdot 2, \varepsilon_{1}=0 \cdot 05$, $\varepsilon_{2}=0.05, c_{1}=0, c_{2}=1.0$ (same for all the three elements)

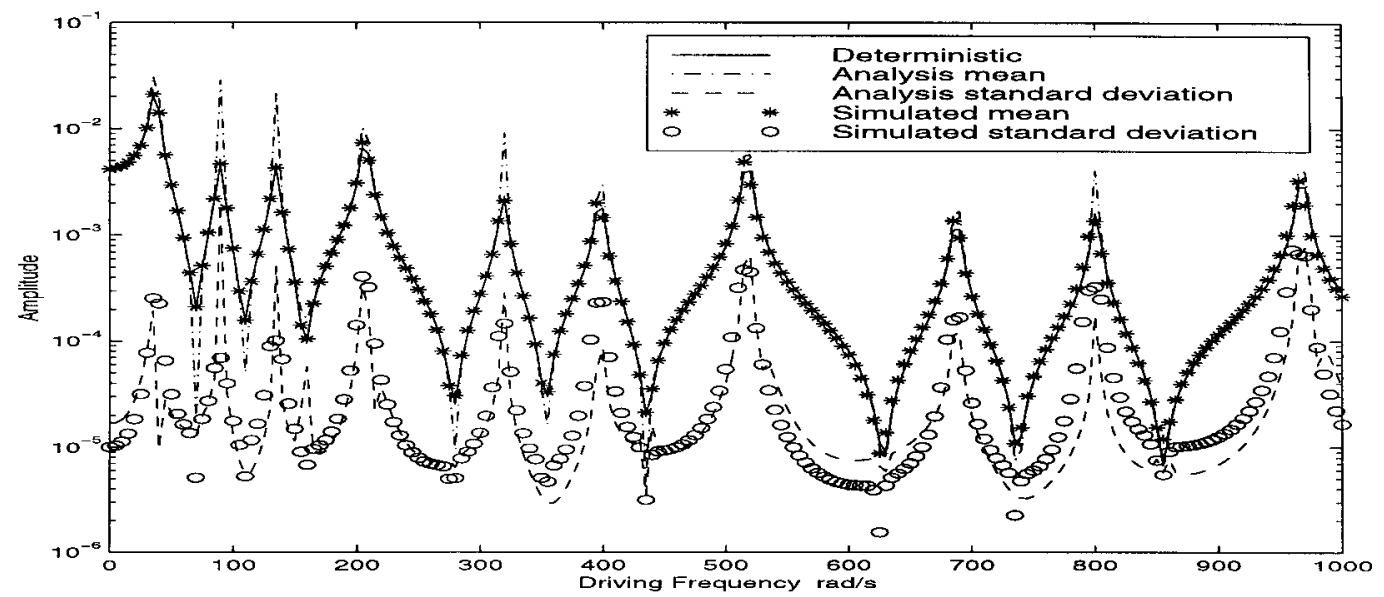

Figure 4. Statistics of amplitude of side sway using eigenfunction expansion method

were written in Fortran 90 and were run on a DEC TuRBoLASER 840 (300 MHz clock speed, $8 \mathrm{~KB}$ Data, $8 \mathrm{~KB}$ Instruction Cache, 4 MB L2 Cache, 2 GB Main Memory, 20 GB Hard Disk) machine. In the numerical work, the computation of covariance matrix of $X_{l}$ was carried out using a two-dimensional, seventh-order Newton-Coates integration scheme. It was observed that the time required for evaluating these integrals constituted about 90 per cent of the computation time for implementing the three theoretical analysis procedures, namely, random eigenfunction expansion method, complex Neumann expansion method and combined analytical and simulation method. Thus, it is estimated that, if properties of distinct elements of the structures are uncorrelated, the computation time is approximately proportional to the number of elements used in the study. Correlation among different elements can be handled within the framework of our approach, but at the cost of increased computational effort. Again, if properties of all the distinct elements of the structure are correlated, the computations time is estimated to increase as $n_{e} \times\left(n_{e}+1\right) / 2$, where $n_{e}$ is the number of elements used. It was also observed that, for the example considered, the time required to run the 100 sample Monte Carlo simulation program is approximately five times more than the programs employing other methods. 


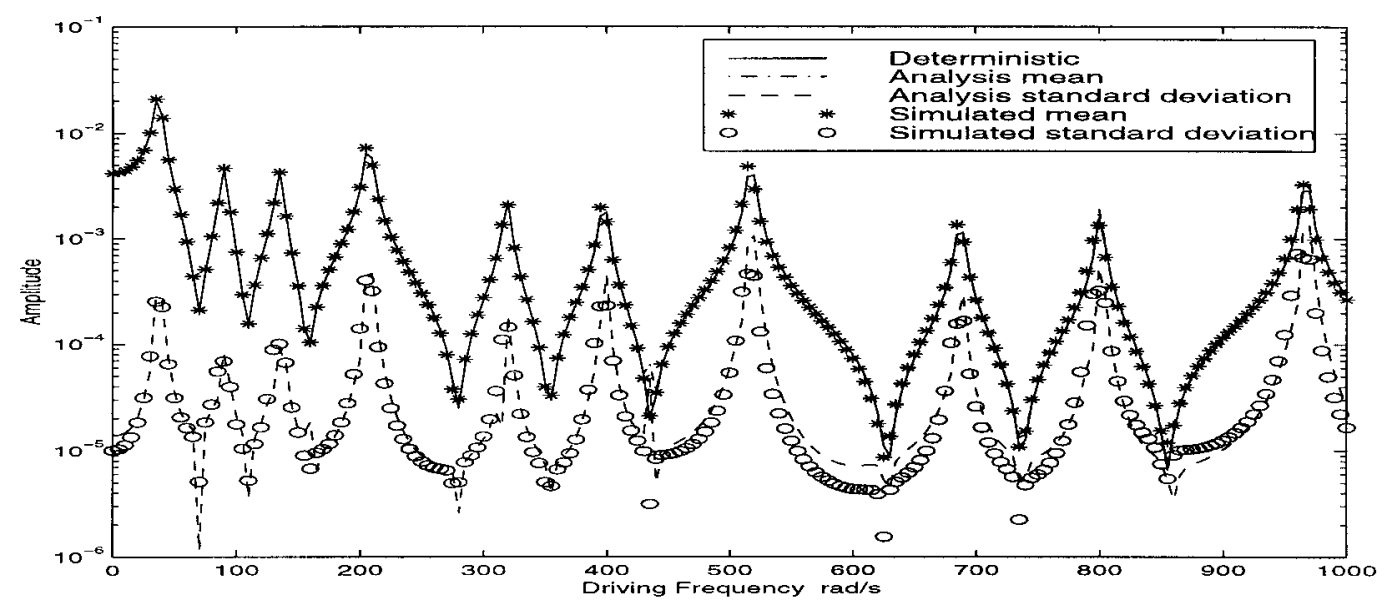

Figure 5. Statistics of amplitude of side sway using Neumann expansion method

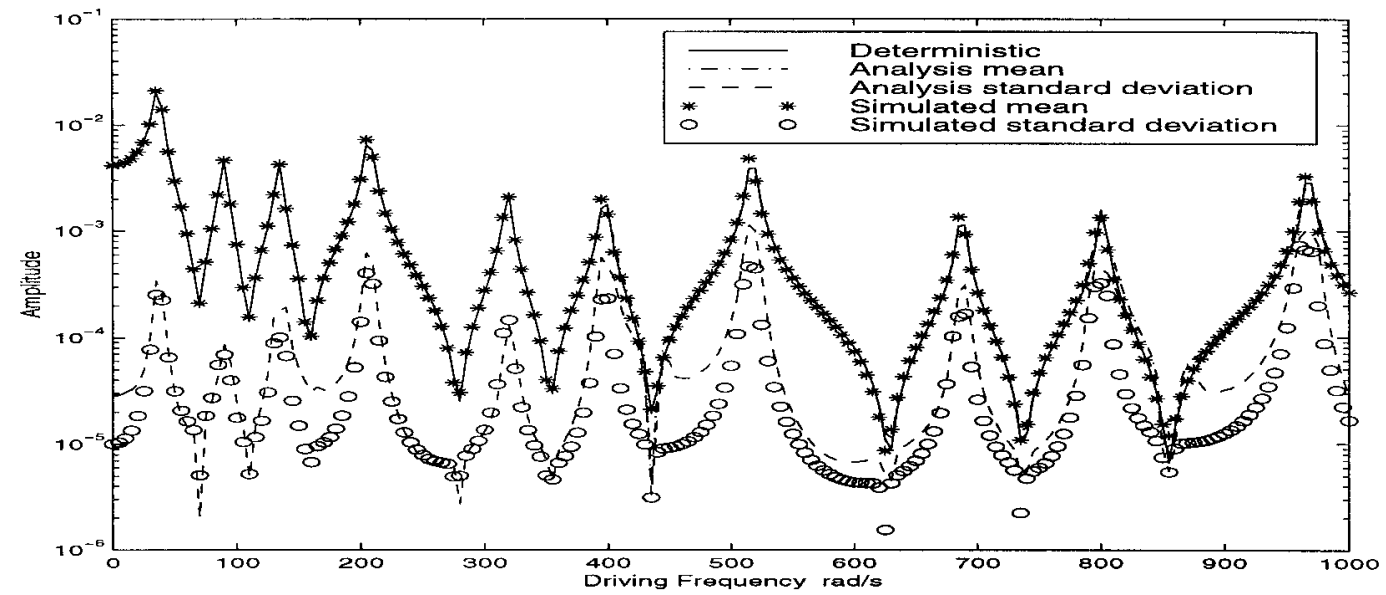

Figure 6. Statistics of amplitude of side sway using combined analytical-simulation method

In producing these numerical results it is assumed that $f_{1}(x)$ and $f_{2}(x)$ are jointly Gaussian random fields. This, as has been already noted, affords a major simplification in applying analytical procedures, since, by virtue of this approximation, together with the assumptions of first order perturbations for random eigenfunction expansion method and one term correction for complex Neumann expansion method, the probability distribution function of the real and imaginary parts of the response vector also becomes Gaussian. This, in turn, enables the calculation of the statistics of the displacement amplitude and phase by a two dimensional integration over a Gaussian probability distribution function. It must however be noted that, as a consequence of the use of Gaussian models for system property random fields, the applicability of the methods developed in this paper gets restricted to small random variations in system properties. Similar restrictions are also imposed by the expansion techniques used in the analytical formulations. 


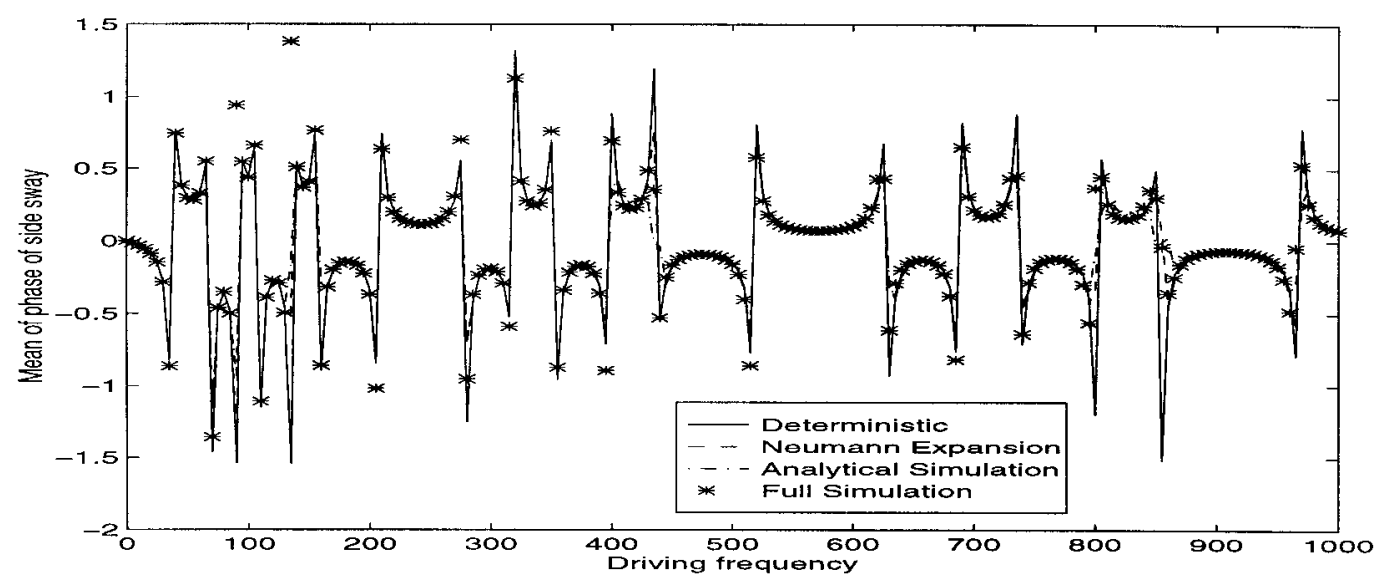

Figure 7. Mean of phase of side sway

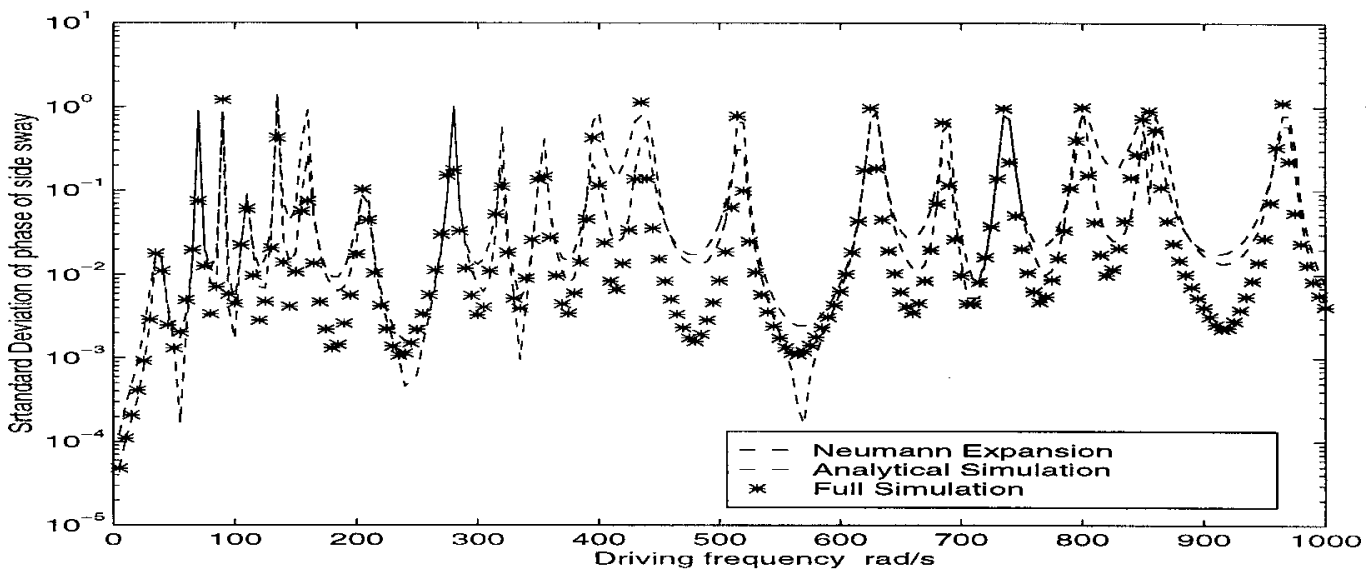

Figure 8. Standard deviation of phase of side sway

In the simulations studies too, the use of Gaussian models for system property random fields can lead to unreasonable situations. Thus, for instance, on account of unbounded nature of the Gaussian variables, it is conceivable that flexural stiffness of the frame members get reduced to zero at distinct points on the frame. Physically, this amounts to formation of plastic hinges in the system and the system would start behaving as a mechanism. Consequently, the sample displacement would become unbounded. Given that the simulation results should asymptotically approach the exact results, we reach the inescable conclusion that the simulated results become unbounded. In our simulation studies we have excluded samples which resulted in negative values for the mass/stiffness processes. This would mean that we are simulating random processes whose first order probability distributions are conditioned Gaussian functions (conditioned to be greater than zero). Strictly speaking, the correlation function of such conditioned processes would not be identical to the correlation functions of the unconditioned Gaussian random process. In this 
case the mean of the random perturbations also would not be strictly zero unless an additional symmetry condition is also imposed. These errors, for the randomness magnitude considered in our studies $(\varepsilon \leqslant 0.05)$ is expected not to be big enough to the extent that they would significantly alter the predictions on response mean and standard deviation. Thus, the anomalous situation resulting from negative stiffness, as has been described above, and a similar problematic situation arising from zero or negative mass, have been excluded from our simulation results, albeit in an imperfect manner.

It is observed from the numerical results that the theoretical predictions generally compare well with the simulations results over the entire frequency range considered. This supports the approximations made in this study in the treatment of system randomness and damping in deriving the element dynamic stiffness matrix and also in inverting the random global dynamic stiffness matrix to calculate the displacement amplitudes. At resonance points, the theory and simulations compare better than at the anti-resonance points. The numerical results on response statistics indicate that the amplitude and phase processes evolve in a nonstationary manner in $\omega$. The mean results are found to closely follow the deterministic results. However, it is observed that in high-frequency ranges the response variability increases, which gets characterized by relatively higher values of standard deviation, especially near the resonant frequency points, where the standard deviation sometimes becomes comparable to the mean value. This is significant, since, the standard deviations of the beam property random fields are only 5 per cent of the corresponding mean values, which would mean that the system dynamics magnifies the structural uncertainties considerably in the high frequency ranges. This fact emphasizes the relevance of the current study, especially in higher driving frequency ranges.

Finally, it must be noted that the frequency-dependent shape functions used in this study provides relief in selection of mesh sizes only with respect to driving frequency. These functions, however, cannot relive the dependence of mesh size on the correlation lengths of the system property random fields. The dependence of mesh size with respect to correlation lengths can be overcome if one uses stochastic shape functions. Such shape functions can seldom be determined exactly. However, approximations to these functions can be obtained by employing approximate methods of analysis of random differential equations, such as those based on closure hypothesis. But this would make the further analysis very complicated. These aspects and similar issues related to selection of mesh sizes with respect to correlation lengths of the random fields have not been addressed in our study.

\section{CONCLUSIONS}

A dynamic weighted integral approach combined with the direct dynamic stiffness matrix method for harmonic vibration analysis of framed structures with statistical uncertainties is studied. The element property random fields are discretized using frequency dependent shape functions. This relaxes the requirements on using finer finite element mesh sizes for higher ranges of driving frequency. The formulation leads to a complex, symmetric, random global dynamic stiffness matrix. Two analytical procedures and one combined analytical and simulation method are discussed for inverting this matrix and their performance is compared with the results of a more exact, but computationally intensive, full scale simulation scheme. These comparisons are demonstrated to be reasonably good over a wide range of driving frequency. The methods discussed herein are particularly advantageous if operating frequency range lies in higher system natural frequency 
ranges and also when several structural modes contribute to the response. Further improvements to the method are possible with respect to the following aspects:

(a) effective numerical methods for inversion of complex symmetric matrices taking the advantage of their banded structure;

(b) inclusion of higher-order terms in complex Neumann expansion method and also in the perturbation approach;

(c) inclusion of non-Gaussian models for random fields $f_{i}(x)$ which satisfy the requirement that $P\left[1+\varepsilon_{i} f_{i}(x)<0\right]=0$.

Work on achieving these improvements and, also in applying the approach to transient dynamic analysis of built up random skeletal structures, is currently being carried out by us.

\section{APPENDIX I}

\section{I.1. Expressions for elements of matrices $\left[\alpha^{l}(\omega)\right]$ appearing in equation (12)}

$$
\begin{aligned}
& \alpha_{1,1}^{1}=\frac{1}{4} \frac{(c S+C S)^{2}}{(-1+c C)^{2}}, \quad \alpha_{1,2}^{1}=\frac{1}{4} \frac{(c S+C s)(-1+c C+s S)}{(-1+c C)^{2} b}, \alpha_{1,3}^{1}=-\frac{1}{4} \frac{(c S+C s)(S+s)}{(-1+c C)^{2}}, \alpha_{1,4}^{1}=-\frac{1}{4} \frac{(c S+C s)(-C+c)}{(-1+c C)^{2} b} \\
& \alpha_{2,2}^{1}=\frac{1}{4} \frac{(-1+c C+s S)^{2}}{b^{2}(-1+c C)^{2}}, \quad \alpha_{2,3}^{1}=-\frac{1}{4} \frac{(-1+c C+s S)(S+s)}{b(-1+c C)^{2}}, \alpha_{2,4}^{1}=-\frac{1}{4} \frac{(-1+c C+s S)(-C+c)}{b^{2}(-1+c C)^{2}}, \alpha_{3,3}^{1}=\frac{1}{4} \frac{(S+s)^{2}}{(-1+c C)^{2}} \\
& \alpha_{3,4}^{1}=\frac{1}{4} \frac{(S+s)(-C+c)}{(-1+c C)^{2} b}, \quad \alpha_{4,4}^{1}=\frac{1}{4} \frac{(-C+c)^{2}}{b^{2}(-1+c C)^{2}} \\
& \alpha_{1,1}^{2}=\frac{1}{2} \frac{(c S+C s)(-s S-1+c C)}{(-1+c C)^{2}}, \quad \alpha_{1,2}^{2}=\frac{1}{4} \frac{c^{2} S^{2}-C^{2} s^{2}-s^{2} S^{2}+1-2 c C+c^{2} C^{2}}{(-1+c C)^{2} b}, \alpha_{1,3}^{2}=-\frac{1}{4} \frac{c^{2} S-C^{2} s+2 C s c-s S^{2}-s^{2} S-S-s}{(-1+c C)^{2}} \\
& \alpha_{1,4}^{2}=-\frac{1}{4} \frac{c S^{2}-2 c S s+2 C s S-C s^{2}+C-c-c C^{2}+c^{2} C}{(-1+c C)^{2} b}, \alpha_{2,2}^{2}=\frac{1}{2} \frac{(-1+c C+s S)(-C s+c S)}{b^{2}(-1+c C)^{2}} \\
& \alpha_{2,3}^{2}=-\frac{1}{4} \frac{C-c-c C^{2}+c^{2} C-2 C s S+2 c S s-C s^{2}+c S^{2}}{(-1+c C)^{2} b}, \alpha_{2,4}^{2}=-\frac{1}{4} \frac{-S+s-2 C s c+s S^{2}-s^{2} S+C^{2} s+c^{2} S}{b^{2}(-1+c C)^{2}} \\
& \alpha_{3,3}^{2}=\frac{1}{2} \frac{(S+s)(-C+c)}{(-1+c C)^{2}}, \alpha_{3,4}^{2}=\frac{1}{4} \frac{S^{2}-s^{2}+C^{2}-2 c C+c^{2}}{(-1+c C)^{2} b}, \alpha_{4,4}^{2}=\frac{1}{2} \frac{(-C+c)(S-s)}{b^{2}(-1+c C)^{2}} \\
& \alpha_{1,1}^{3}=-\frac{1}{2} \frac{(c S+C s)^{2}}{(-1+c C)^{2}}, \quad \alpha_{1,2}^{3}=-\frac{1}{2} \frac{(c S+C s) s S}{(-1+c C)^{2} b}, \quad \alpha_{1,3}^{3}=\frac{1}{2} \frac{(c S+C s)(S+s)}{(-1+c C)^{2}}, \quad \alpha_{1,4}^{3}=\frac{1}{2} \frac{(c S+C s)(-C+c)}{(-1+c C)^{2} b} \\
& \alpha_{2,2}^{3}=\frac{1}{2} \frac{(-s S-1+c C)(-1+c C+s S)}{b^{2}(-1+c C)^{2}}, \alpha_{2,3}^{3}=\frac{1}{2} \frac{(S+s) s S}{(-1+c C)^{2} b}, \quad \alpha_{2,4}^{3}=\frac{1}{2} \frac{(-C+c) s S}{b^{2}(-1+c C)^{2}}, \quad \alpha_{3,3}^{3}=-\frac{1}{2} \frac{(S+s)^{2}}{(-1+c C)^{2}} \\
& \alpha_{3,4}^{3}=-\frac{1}{2} \frac{(S+s)(-C+c)}{(-1+c C)^{2} b}, \alpha_{4,4}^{3}=-\frac{1}{2} \frac{(-C+c)^{2}}{b^{2}(-1+c C)^{2}} \\
& \alpha_{1,1}^{4}=\frac{1}{2} \frac{(c S+C s)(-1+c C+s S)}{(-1+c C)^{2}}, \alpha_{1,2}^{4}=-\frac{1}{4} \frac{c^{2} S^{2}-C^{2} s^{2}-1+2 c C+2 s S-c^{2} C^{2}-2 c S C s-s^{2} S^{2}}{(-1+c C)^{2} b} \\
& \alpha_{1,3}^{4}=\frac{1}{4} \frac{-2 c S C+c^{2} S-C^{2} s+S+s-s S^{2}-s^{2} S}{(-1+c C)^{2}}, \alpha_{1,4}^{4}=-\frac{1}{4} \frac{-c S^{2}+2 c S s-2 C s S+C s^{2}+C-c-c C^{2}+c^{2} C}{\left(-1+c C C^{2} b\right.} \\
& \alpha_{2,2}^{4}=-\frac{1}{2} \frac{(-1+c C+s S)(-C s+c S)}{b^{2}(-1+c C)^{2}}, \alpha_{2,3}^{4}=\frac{1}{4} \frac{C-c-c C^{2}+c^{2} C-2 C s S+2 c S s-C s^{2}+c S^{2}}{(-1+c C)^{2} b} \\
& \alpha_{2,4}^{4}=\frac{1}{4} \frac{-S+s-2 C s c+s S^{2}-s^{2} S+C^{2} s+c^{2} S}{b^{2}(-1+c C)^{2}}, \alpha_{3,3}^{4}=-\frac{1}{2} \frac{(S+s)(-C+c)}{(-1+c C)^{2}}, \alpha_{3,4}^{4}=-\frac{1}{4} \frac{S^{2}-s^{2}+C^{2}-2 c C+c^{2}}{(-1+c C)^{2} b} \\
& \alpha_{4,4}^{4}=-\frac{1}{2} \frac{(-C+c)(S-s)}{b^{2}(-1+c C)^{2}} \\
& \alpha_{1,1}^{5}=\frac{1}{4} \frac{(-s S-1+c C)^{2}}{(-1+c C)^{2}}, \quad \alpha_{1,2}^{5}=\frac{1}{4} \frac{(-s S-1+c C)(-C s+c S)}{(-1+c C)^{2} b}, \alpha_{1,3}^{5}=-\frac{1}{4} \frac{(-s S-1+c C)(-C+c)}{(-1+c C)^{2}} \\
& \alpha_{1,4}^{5}=-\frac{1}{4} \frac{(-s S-1+c C)(S-s)}{(-1+c C)^{2} b}, \alpha_{2,2}^{5}=\frac{1}{4} \frac{(-C s+c S)^{2}}{b^{2}(-1+c C)^{2}}, \quad \alpha_{2,3}^{5}=-\frac{1}{4} \frac{(-C s+c S)(-C+c)}{(-1+c C)^{2} b}, \alpha_{2,4}^{5}=-\frac{1}{4} \frac{(-C s+c S)(S-s)}{b^{2}(-1+c C)^{2}} \\
& \alpha_{3,3}^{5}=\frac{1}{4} \frac{(-C+c)^{2}}{(-1+c C)^{2}}, \quad \alpha_{3,4}^{5}=\frac{1}{4} \frac{(-C+c)(S-s)}{(-1+c C)^{2} b}, \quad \alpha_{4,4}^{5}=\frac{1}{4} \frac{(S-s)^{2}}{b^{2}(-1+c C)^{2}}
\end{aligned}
$$




$$
\begin{aligned}
& \alpha_{1,1}^{6}=-\frac{1}{2} \frac{(c S+C s)(-s S-1+c C)}{(-1+c C)^{2}}, \alpha_{1,2}^{6}=-\frac{1}{4} \frac{\left(-s^{2} S^{2}-2 s S+2 c S C s-1+2 c C-c^{2} C^{2}+c^{2} S^{2}-C^{2} s^{2}\right)}{(-1+c C)^{2} b} \\
& \alpha_{1,3}^{6}=\frac{1}{4} \frac{c^{2} S-C^{2} s+2 C s c-s S^{2}-s^{2} S-S-s}{(-1+c C)^{2}}, \alpha_{1,4}^{6}=\frac{1}{4} \frac{c S^{2}-2 c S s+2 C s S-C s^{2}+C-c-c C^{2}+c^{2} C}{(-1+c C)^{2} b} \\
& \alpha_{2,2}^{6}=\frac{1}{2} \frac{(-s S-1+c C)(-C s+c S)}{b^{2}(-1+c C)^{2}}, \alpha_{2,3}^{6}=-\frac{1}{4} \frac{2 C s S+C s^{2}-c S^{2}-2 c S s+C-c-c C^{2}+c^{2} C}{(-1+c C)^{2} b} \\
& \alpha_{2,4}^{6}=\frac{1}{4} \frac{C^{2} s-2 c S C+c^{2} S+s S^{2}-s^{2} S+S-s}{b^{2}(-1+c C)^{2}}, \alpha_{3,3}^{6}=-\frac{1}{2} \frac{(S+s)(-C+c)}{(-1+c C)^{2}}, \alpha_{3,4}^{6}=-\frac{1}{4} \frac{S^{2}-s^{2}+C^{2}-2 c C+c^{2}}{(-1+c C)^{2} b} \\
& \alpha_{4,4}^{6}=-\frac{1}{2} \frac{(-C+c)(S-s)}{b^{2}(-1+c C)^{2}} \\
& \alpha_{1,1}^{7}=\frac{1}{2} \frac{(-s S-1+c C)(-1+c C+s S)}{(-1+c C)^{2}}, \quad \alpha_{1,2}^{7}=\frac{1}{2} \frac{(-C s+c S) s S}{(-1+c C)^{2} b}, \quad \alpha_{1,3}^{7}=-\frac{1}{2} \frac{(-C+c) s S}{(-1+c C)^{2}}, \quad \alpha_{1,4}^{7}=-\frac{1}{2} \frac{(S-s) s S}{(-1+c C)^{2} b} \\
& \alpha_{2,2}^{7}=-\frac{1}{2} \frac{(-C s+c S)^{2}}{b^{2}(-1+c C)^{2}}, \quad \alpha_{2,3}^{7}=\frac{1}{2} \frac{(-C s+c S)(-C+c)}{(-1+c C)^{2} b}, \quad \alpha_{2,4}^{7}=\frac{1}{2} \frac{(-C s+c S)(S-s)}{b^{2}(-1+c C)^{2}}, \quad \alpha_{3,3}^{7}=-\frac{1}{2} \frac{(-C+c)^{2}}{(-1+c C)^{2}} \\
& \alpha_{3,4}^{7}=-\frac{1}{2} \frac{(-C+c)(S-s)}{(-1+c C)^{2} b}, \alpha_{4,4}^{7}=-\frac{1}{2} \frac{(S-s)^{2}}{b^{2}(-1+c C)^{2}} \\
& \alpha_{1,1}^{8}=\frac{1}{4} \frac{(c S+C s)^{2}}{(-1+c C)^{2}}, \quad \alpha_{1,2}^{8}=-\frac{1}{4} \frac{(c S+C s)(-s S-1+c C)}{(-1+c C)^{2} b}, \alpha_{1,3}^{8}=-\frac{1}{4} \frac{(c S+C s)(S+s)}{(-1+c C)^{2}}, \alpha_{1,4}^{8}=-\frac{1}{4} \frac{(c S+C s)(-C+c)}{(-1+c C)^{2} b} \\
& \alpha_{2,2}^{8}=\frac{1}{4} \frac{(-s S-1+c C)^{2}}{b^{2}(-1+c C)^{2}}, \alpha_{2,3}^{8}=\frac{1}{4} \frac{(-s S-1+c C)(S+s)}{(-1+c C)^{2} b}, \alpha_{2,4}^{8}=\frac{1}{4} \frac{(-s S-1+c C)(-C+c)}{b^{2}(-1+c C)^{2}}, \alpha_{3,3}^{8}=\frac{1}{4} \frac{(S+s)^{2}}{(-1+c C)^{2}} \\
& \alpha_{3,4}^{8}=\frac{1}{4} \frac{(S+s)(-C+c)}{(-1+c C)^{2} b}, \alpha_{4,4}^{8}=\frac{1}{4} \frac{(-C+c)^{2}}{b^{2}(-1+c C)^{2}} \\
& \alpha_{1,1}^{9}=-\frac{1}{2} \frac{(c S+C s)(-1+c C+s S)}{(-1+c C)^{2}}, \alpha_{1,2}^{9}=\frac{1}{4} \frac{c^{2} S^{2}-C^{2} s^{2}-s^{2} S^{2}+1-2 c C+c^{2} C^{2}}{(-1+c C)^{2} b} \\
& \alpha_{1,3}^{9}=-\frac{1}{4} \frac{-2 c S C+c^{2} S-C^{2} s+S+s-s S^{2}-s^{2} S}{(-1+c C)^{2}}, \alpha_{1,4}^{9}=\frac{1}{4} \frac{-c S^{2}+2 c S s-2 C S S+C s^{2}+C-c-c C^{2}+c^{2} C}{(-1+c C)^{2} b} \\
& \alpha_{2,2}^{9}=-\frac{1}{2} \frac{(-s S-1+c C)(-C S+c S)}{b^{2}(-1+c C)^{2}}, \alpha_{2,3}^{9}=\frac{1}{4} \frac{2 C s S+C s^{2}-c S^{2}-2 c S s+C-c-c C^{2}+c^{2} C}{(-1+c C)^{2} b} \text {, } \\
& \alpha_{2,4}^{9}=-\frac{1}{4} \frac{C^{2} s-2 c S C+c^{2} S+s S^{2}-s^{2} S+S-s}{b^{2}(-1+c C)^{2}}, \alpha_{3,3}^{9}=\frac{1}{2} \frac{(S+s)(-C+c)}{(-1+c C)^{2}}, \alpha_{3,4}^{9}=\frac{1}{4} \frac{S^{2}-s^{2}+C^{2}-2 c C+c^{2}}{(-1+c C)^{2} b} \\
& \alpha_{4,4}^{9}=\frac{1}{2} \frac{(-C+c)(S-s)}{b^{2}(-1+c C)^{2}} \text {, } \\
& \alpha_{1,1}^{10}=\frac{1}{4} \frac{(-1+c C+s S)^{2}}{(-1+c C)^{2}}, \quad \alpha_{1,2}^{10}=-\frac{1}{4} \frac{(-1+c C+s S)(-C s+c S)}{(-1+c C)^{2} b}, \alpha_{1,3}^{10}=\frac{1}{4} \frac{(-1+c C+s S)(-C+c)}{(-1+c C)^{2}} \\
& \alpha_{1,4}^{10}=\frac{1}{4} \frac{(-1+c, C+s S)(S-s)}{(-1+c C)^{2} b}, \quad \alpha_{2,2}^{10}=\frac{1}{4} \frac{(-C s+c S)^{2}}{b^{2}(-1+c C)^{2}}, \quad \alpha_{2,3}^{10}=-\frac{1}{4} \frac{(-C s+c S)(-C+c)}{(-1+c C)^{2} b}, \alpha_{2,4}^{10}=-\frac{1}{4} \frac{(-C s+c S)(S-s)}{b^{2}(-1+c C)^{2}} \\
& \alpha_{3,3}^{10}=\frac{1}{4} \frac{(-C+c)^{2}}{(-1+c C)^{2}}, \quad \alpha_{3,4}^{10}=\frac{1}{4} \frac{(-C+c)(S-s)}{(-1+c C)^{2} b}, \quad \alpha_{4,4}^{10}=\frac{1}{4} \frac{(S-s)^{2}}{b^{2}(-1+c C)^{2}}
\end{aligned}
$$

In the above expressions $C=\cosh b l, c=\cos b l, S=\sinh b l, s=\sin b l$ and

$$
b^{4}=\frac{m_{0} \omega^{2}}{\mathrm{EI}_{0}}
$$

It may be noted that the matrices $\left[\boldsymbol{\alpha}^{l}(\omega)\right]$ are all symmetric and only the upper triangle part of these matrices have been listed above.

\section{APPENDIX II}

\section{II.1. Statistics of random basis vectors}

The eigenvalue problem that we are interested in solving is given by

$$
\left[\mathbf{K}_{\mathrm{R}}^{0}+\Delta \mathbf{K}\right]^{g} \phi=\lambda_{g}{ }^{g} \phi
$$


We begin by introducing the transformation

$$
{ }^{g} \phi=\mathbf{C}^{g} \mathbf{y}
$$

where $\mathbf{C}$ is a matrix of normalized eigenvectors of the deterministic matrix $\mathbf{K}_{\mathrm{R}}^{0}$. Substituting ${ }^{g} \phi$ in equation (55) and premultiplying by $\mathbf{C}^{\mathrm{t}}$ we get

$$
\mathbf{C}^{\mathrm{t}}\left[\mathbf{K}_{\mathrm{R}}^{0}+\Delta \mathbf{K}\right] \mathbf{C}^{g} \mathbf{y}=\lambda_{g}\left[\mathbf{C}^{\mathrm{t}} \mathbf{C}\right]^{g} \mathbf{y} \quad \text { or } \quad\left[\hat{\mathbf{K}}_{\mathrm{r}}^{0}+\Delta \hat{\mathbf{K}}\right]^{g} \mathbf{y}=\lambda_{g}{ }^{g} \mathbf{y}
$$

where

$$
\hat{K}_{i j}^{0}=\delta_{i j} \mu_{i} \quad \text { and } \quad \Delta \hat{K}_{i j}=\sum_{r=1}^{n} \sum_{s=1}^{n} C_{r i} C_{s j} \Delta K_{i j}
$$

The eigenvalues $\lambda_{g}$ and the eigenvectors ${ }^{g} \mathbf{y}$ of the modified problem (57) can now be determined from the system of random linear equations

$$
\left[\hat{\mathbf{K}}_{\mathrm{R}}^{0}+\Delta \hat{\mathbf{K}}-\mathbf{I} \boldsymbol{\lambda}_{g}\right]^{g} \mathbf{y}=0
$$

in which $\mathbf{I}$ denotes the identity matrix. The eigenvectors ${ }^{g} \mathbf{y}$ are normalized by using the condition $\left({ }^{g} \mathbf{y},{ }^{g} \mathbf{y}\right)=1$. For the eigenvalues $\lambda_{g}$ and normalized eigenvectors ${ }^{g} \mathbf{y}$, we assume that the expansions

$$
\lambda_{g}=\mu_{g}-\sum_{p=1}^{\infty} \lambda_{g p}, \quad{ }^{g} \mathbf{y}=\sum_{p=0}^{\infty}{ }^{g} \mathbf{y}_{p}
$$

exist, where $\mu_{g}$ are the eigenvalues of the matrix $\mathbf{K}_{\mathrm{R}}^{0}$ and ${ }^{g} \mathbf{y}_{0}$ is the $g$ th normalized eigenvector with all its elements equal to zero except the $g$ th element which is set to be equal to one. The terms $\lambda_{g p}$ and ${ }^{g} \mathbf{y}_{p}$ denotes the $p$ th-order perturbation terms of $\lambda_{g}$ and ${ }^{g} \mathbf{y}$, respectively. In the present study, we focus our attention on solution only up to the first order and, consequently, following the standard perturbation formalism, we get

$$
\begin{array}{ll}
\text { for } p=0: & \left(\hat{\mathbf{K}}_{\mathrm{R}}^{0}-\mu_{g} \mathbf{I}\right)^{g} \mathbf{y}_{0}=0 \\
\text { for } p=1: & \left(\hat{\mathbf{K}}_{\mathrm{R}}^{0}-\mu_{g} \mathbf{I}\right)^{g} \mathbf{y}_{1}=-\left(\Delta \hat{\mathbf{K}}+\lambda_{g 1} \mathbf{I}\right)^{g} \mathbf{y}_{0}
\end{array}
$$

Equation (61) is satisfied by the chosen ${ }^{g} \mathbf{y}_{0}$. However, ${ }^{g} \mathbf{y}_{1}$ cannot be directly obtained from equation (62) since $\left(\hat{\mathbf{K}}_{\mathrm{R}}^{0}-\mu_{g} \mathbf{I}\right)^{-1}$ does not exist. Now taking the scalar product ${ }^{g} \mathbf{y}_{0}$ to the righthand side of equation (62) we get

$$
\left(\left[\Delta \hat{\mathbf{K}}+\lambda_{g 1} \mathbf{I}\right]{ }^{g} \mathbf{y}_{0},{ }^{g} \mathbf{y}_{0}\right)=0 \quad \text { or } \quad \lambda_{g 1}=-\Delta \hat{K}_{g g}
$$

To find out the eigenvector ${ }^{g} \mathbf{y}_{1}$, arbitrary parameter in the $g$ th component of ${ }^{g} \mathbf{y}_{1}$ is set to zero. Now using equation (62), vector ${ }^{g} \mathbf{y}_{1}$ can be determined and its $i$ th component is expressed as

$$
{ }^{g} \mathbf{y}_{1_{i}}=-\frac{\Delta \hat{K}_{i g}}{\mu_{i g}} \quad \text { for } i \neq g ; \quad{ }^{g} \mathbf{y}_{1_{i}}=0 \quad \text { for } i=g
$$

where $\mu_{i g}=\mu_{i}-\mu_{g}$. Now substituting the expression of $\lambda_{g 1}$ from equation (63) and ${ }^{g} \mathbf{y}_{1_{i}}$ from equation (64) into equation (60), the expression of random eigenvalue and random eigenvectors of the modified problem (equation (57)) can be written as

$$
\lambda_{g}=\mu_{g}+\Delta \hat{K}_{g g}, \quad{ }^{g} \mathbf{y}_{i}={ }^{g} \mathbf{y}_{0_{i}}-\frac{\Delta \hat{K}_{i g}}{\mu_{i g}} \quad \text { for } i \neq g \quad \text { and } \quad{ }^{g} \mathbf{y}_{i}=1 \quad \text { for } i=g
$$


with

$$
\left\langle\lambda_{g}\right\rangle=\mu_{g} \quad \text { and } \quad\left\langle\mathbf{y}_{i}\right\rangle=\delta_{i g}
$$

The correlation of the $i$ th element of $g$ th eigenvector and $j$ th element of $h$ th eigenvector, denoted by $R_{i j}^{g h}$, can now be expressed as

$$
R_{i j}^{g h}=\left(1-\delta_{i g}\right)\left(1-\delta_{j h}\right) \frac{1}{\mu_{i g} \mu_{g h}}\left[\left\langle\Delta \hat{K}_{i g} \Delta \hat{K}_{j h} i\right\rangle\right]
$$

Furthermore, the statistics of the eigenvectors of the original problem ${ }^{g} \phi$, see equation (55), can be related to $R_{i j}^{g h}$ following equation (56) and can be shown to be given by equation (37).

\section{ACKNOWLEDGEMENTS}

This work has been supported by funding from the Department of Science and Technology, Government of India. We thank the reviewers of this paper for their insightful comments.

\section{REFERENCES}

1. R. W. Clough and J. Penzien, Dynamics of Structures, 2nd edn, McGraw-Hill, New York, 1993.

2. E. C. Pestel and F. A. Leckie, Matrix Methods in Elastomechanics, McGraw-Hill, New York, 1963.

3. J. F. Doyle, Wave Propagation in Structures, Springer, New York, 1989.

4. R. A. Ibrahim, 'Structural dynamics with parameter uncertainties', Appl. Mech. Rev., 40(3), 309-328 (1987) .

5. M. Shinozuka and F. Yamazaki, 'Stochastic finite element analysis: an introduction', in by S. T. Ariaratnam, G. I. Schueller and I. Elishakoff (eds.), Stochastic Structural Dynamics: Progress in Theory and Applications, Elsevier, Applied Science, London, 1988.

6. W. K. Liu, T. Belytschko and Y. J. Lua, 'Stochastic computational mechanics for aerospace structures', Prog. Aeronaut. Astronaut. AIAA (1992).

7. H. Benaroya, 'Random eigenvalues, algebraic methods and structural dynamic models', Appl. Math. Comput., 52, 37-66 (1992).

8. H. Benaroya and M. Rehak, 'Finite element methods in probabilistic structural analysis: a selective review', Appl. Mech. Rev., 41, 201-213 (1988).

9. C. E. Brenner, 'Stochastic finite elements (Literature review)', Internal working report No. 35-91, Institute of Engineering Mechanics, University of Innsbruck, Austria, 1991.

10. R. Ghanem and P. D. Spanos, Stochastic Finite Elements: a Spectral Approach, Springer, Berlin, 1991.

11. M. Kleiber and T. D. Hien, The Stochastic Finite Element Method, Wiley, Chichester, 1992.

12. A. Der Kiureghian, C. C. Li and Y. Zhang, 'Recent developments in stochastic finite elements', in R. Rackwitz and P. Thoft-Christensen (eds.), Proc. 4th IFIP WG 7.5 Conf., Munich, Germany, 11-13 September, 1991.

13. Y. K. Lin and G. Q. Cai, Probabilistic Structural Dynamics, McGraw-Hill, New York, 1995.

14. M. Shinozuka, 'Structural response variability', J. Engng. Mech. ASCE, 113(6), 825-842 (1987).

15. G. Deodatis, 'Weighted integral method I: stochastic stiffness matrix', J. Engng. Mech. ASCE, 117, 1851-1864 (1991).

16. T. Takada, 'Weighted integral method in stochastic finite element analysis', Probab. Engng. Mech., 5(3), 146-156 (1990).

17. T. Takada, 'Weighted integral method in multidimensional stochastic finite element analysis', Probab. Engng. Mech., 5(4), 158-166 (1990).

18. C. G. Bucher and C. E. Brenner, 'Stochastic response of uncertain systems', Arch. Appl. Mech., 62, 507-516 (1992).

19. C. S. Manohar and S. Adhikari, 'Dynamic stiffness of randomly parametered beams', Prob. Engng. Mech., 13(1), 39-51 (1998).

20. O. C. Zienkiewicz and K. Morgan, The Finite Elements and Approximation, Wiley, New York, 1982.

21. J. V. Scheidt and W. Purkert, Random Eigenvalue Problems, North-Holland, New York, 1983.

22. A. Sarkar and C. S. Manohar, 'Dynamic stiffness of a general cable element', Arch. Appl. Mech., 66, 315-325 (1996). 